PREPARED FOR THE U.S. DEPARTMENT OF ENERGY, UNDER CONTRACT DE-AC02-76CH03073

PPPL-3650

PPPL-3650

UC-70

Mechanisms of Stochastic Diffusion of Energetic lons in Spherical Tori

by

Ya.I. Kolesnichenko, R.B. White, and Yu.V. Yakovenko

January 2002

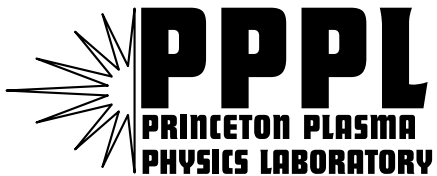

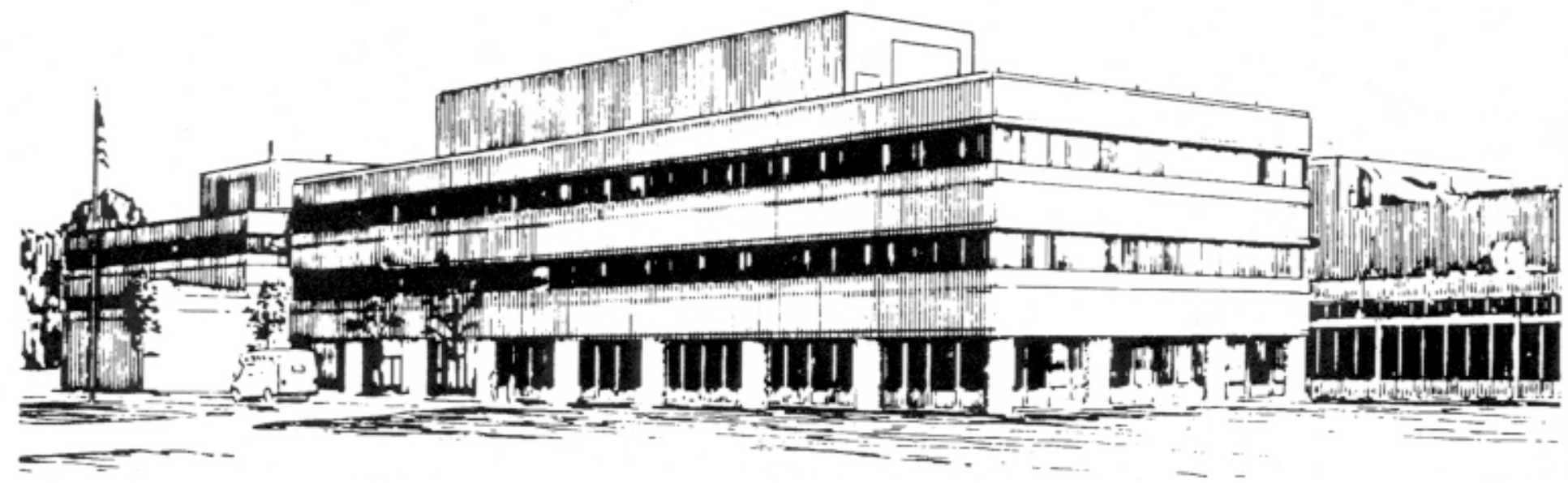

PRINCETON PLASMA PHYSICS LABORATORY PRINCETON UNIVERSITY, PRINCETON, NEW JERSEY 


\section{PPPL Reports Disclaimer}

This report was prepared as an account of work sponsored by an agency of the United States Government. Neither the United States Government nor any agency thereof, nor any of their employees, makes any warranty, express or implied, or assumes any legal liability or responsibility for the accuracy, completeness, or usefulness of any information, apparatus, product, or process disclosed, or represents that its use would not infringe privately owned rights. Reference herein to any specific commercial product, process, or service by trade name, trademark, manufacturer, or otherwise, does not necessarily constitute or imply its endorsement, recommendation, or favoring by the United States Government or any agency thereof. The views and opinions of authors expressed herein do not necessarily state or reflect those of the United States Government or any agency thereof.

\section{Availability}

This report is posted on the U.S. Department of Energy's Princeton Plasma Physics Laboratory Publications and Reports web site in Fiscal Year 2002. The home page for PPPL Reports and Publications is: http://www.pppl.gov/pub_report/

DOE and DOE Contractors can obtain copies of this report from:

U.S. Department of Energy

Office of Scientific and Technical Information

DOE Technical Information Services (DTIS)

P.O. Box 62

Oak Ridge, TN 37831

Telephone: (865) 576-8401

Fax: (865) 576-5728

Email: reports@adonis.osti.gov

This report is available to the general public from:

National Technical Information Service

U.S. Department of Commerce

5285 Port Royal Road

Springfield, VA 22161

Telephone: 1-800-553-6847 or

(703) 605-6000

Fax: (703) 321-8547

Internet: http://www.ntis.gov/ordering.htm 


\title{
Mechanisms of stochastic diffusion of energetic ions in spherical tori
}

\author{
Ya. I. Kolesnichenko ${ }^{1}$, R. B. White ${ }^{2}$, and Yu. V. Yakovenko ${ }^{1}$ \\ ${ }^{1}$ Institute for Nuclear Research, Prospekt Nauky 47, Kyiv 03680, Ukraine \\ 2 Princeton Plasma Physics Laboratory, P.O. Box 451, Princeton, NJ 08543, USA
}

(January 13, 2002)

\begin{abstract}
Stochastic diffusion of the energetic ions in spherical tori is considered. The following issues are addressed: (i) Goldston-White-Boozer diffusion in a rippled field; (ii) cyclotron-resonance-induced diffusion caused by the ripple; (iii) effects of non-conservation of the magnetic moment in an axisymmetric field. It is found that the stochastic diffusion in spherical tori with a weak magnetic field has a number of peculiarities in comparison with conventional tokamaks; in particular, it is characterized by an increased role of mechanisms associated with non-conservation of the particle magnetic moment. It is concluded that in current experiments on National Spherical Torus eXperiment (NSTX) the stochastic diffusion does not have a considerable influence on the confinement of energetic ions.
\end{abstract}




\section{INTRODUCTION}

The magnetic confinement of charged particles depends on the ability of magnetic configurations to provide integrable particle motion. In axisymmetric toroidal plasmas most particle orbits are regular and weakly deflect from the flux surfaces. However, the motion of some particles may be stochastic, which can lead to the escape of these particles to the wall for a time small in comparison with characteristic collisional times. The stochasticity results from small but chaotic changes of adiabatic invariants of the particle motion caused the presence of the ripple of the toroidal magnetic field, the plasma turbulence, and the finite Larmor radius of the particles.

In particular, the ripple is known to deteriorate the confinement of the energetic ions in Conventional Tokamaks (CT), the dominant loss mechanism being the stochastic diffusion of trapped particles discovered by Goldston, White, and Boozer (GWB). ${ }^{1}$ The ripple amplitude, $\delta_{\text {rip }}$, in Spherical Tori $(\mathrm{ST})$ is of the same order as in CTs; nevertheless, one can expect that the physics of the ripple-induced transport of energetic ions in STs may considerably differ from that in CTs. The matter is that STs are characterized by lower magnetic field, higher $\beta$ ( $\beta$ is the ratio of the plasma pressure to the magnetic field pressure), lower aspect ratio of the torus, and larger Shafranov shift. These factors influence the interaction between the ions and the ripple by both changing the characteristic frequencies of the particle motion (the bounce/transit time frequency, the precession frequency, and the gyrofrequency) and affecting the contours of constant $\delta_{\text {rip }}$. In addition, they can make effects of the fast-ion Larmor radius considerable. Because the magnetic field in STs is much lower than that in CTs, at least, in present-day machines, resonances involving the gyrofrequency of the energetic ions may become of importance. One can expect that specific features of the diffusion appear also because the magnetic field strength in STs can have a maximum near the outer edge of the torus (in high- $\beta$ discharges).

The GWB diffusion arises from the non-conservation of the toroidal angular momentum of a particle, which is a direct consequence of the violation of the axial symmetry by the ripple. In contrast to this, the diffusion associated with the cyclotron resonance be- 
tween the energetic ions and the ripple is caused by simultaneous changes of the toroidal angular momentum and the magnetic moment of the ions. Both circulating and trapped particles rather than trapped ones only can be affected by the ripple through the cyclotron resonance. Attention to possible influence of this resonance on the ripple-induced diffusion of the energetic ions was drawn in Ref. ${ }^{2}$, where, however, it was found that the considered diffusion plays a minor role. In STs, this diffusion was not considered yet.

Probably, the stochastic diffusion of the energetic ions may occur also in axisymmetric laminar plasmas. The matter is that the particle magnetic moment experiences a small but noticeable kick in the point of the orbit where the magnetic field strength is minimum. This effect was discovered more than 40 years ago when studying the particle confinement in the open mirror traps by Garren et al. ${ }^{3}$ Therefore, we will refer to it as "Garren effect".

The purpose of this work is to consider various mechanisms of stochastic diffusion of energetic ions in STs in the absence of MHD activity and to evaluate possible influence of such diffusion on the confinement of the energetic ions, first of all, in the NSTX spherical torus. The structure of the work is as follows. In Sec. II the particle motion is analyzed in the assumption that the stochasticity conditions are satisfied, the analysis being qualitative to a considerable extent. Both the GWB diffusion and the CyclotronResonance-Induced (CRI) diffusion are considered, the analysis employing the similarity of the ripple and a wave perturbation. In addition, the Garren jumps of the magnetic moment and the particle radial coordinate are investigated. In Sec. III a Lagrangian formalism is suggested for the description of the nonadiabaticity of the particle motion caused by the ripple and the Garren effect. In Sec. IV the developed formalism is applied to the derivation of a formula for the Garren change of the magnetic moment. In Sec. V the transition to chaos is investigated, and the critical ripple amplitude is calculated with taking into account the Larmor radius of energetic ions and analyzed for the GWB diffusion and CRI diffusion. This is done by using a new adiabatic invariant of the particle motion. In Sec. VI possible consequences of the stochastic diffusion are discussed. Sec. VII summarizes the obtained results. In Appendix A an expression for the field line curvature in a finite-pressure plasma is derived. Appendix B describes the averaging of 
the particle motion near a resonance, which results in a new adiabatic invariant of motion used in Sec. V.

\section{INTERPLAY OF THE RESONANCES AND PARTICLE MOTION DURING STOCHASTIC DIFFUSION}

We begin with an analysis treating the ripple of the magnetic field as a wave of small amplitude with zero frequency. In order to analyze the effect of the ripple on energetic ions, we consider the kinetic response of these ions. For the sake of simplicity, we assume that the magnetic perturbation, $\tilde{B}$, varies only along the large azimuth of the torus and is characterized by the longitudinal wave number $k_{\|}=N / R_{0}$, where $R_{0}$ is the radius of the magnetic axis of the torus, $N$ is the number of the toroidal field coils. Taking $\tilde{B} \propto \exp (-i N \varphi)$ with $\varphi$ the toroidal angle variable, we write a well-known solution of the linearized kinetic equation for the disturbed part of the particle distribution function $(\tilde{f})$ as follows, cf. Ref. ${ }^{4}$ :

$$
\tilde{f}=\sum_{l s} \mathcal{H} \delta\left(l\left\langle\omega_{B}\right\rangle-s \omega_{b}-N\langle\dot{\varphi}\rangle\right) \tilde{X} \hat{\Pi} F
$$

where

$$
\begin{gathered}
\mathcal{H}=\oint d t \exp \left\{-i \int_{0}^{t} d t^{\prime}\left(l \omega_{B}-N \dot{\varphi}\right)\right\} \\
\hat{\Pi}=\frac{l \omega_{B}}{B} \frac{\partial}{\partial \mu}-N \frac{\partial}{\partial P}
\end{gathered}
$$

$F$ is the unperturbed part of the distribution function, $P=M v_{\|} R-(e / c) \psi$ is the canonical angular momentum, $\psi$ is the poloidal magnetic flux, $R$ is the distance from the major axis of the torus, $v_{\|}$is the particle velocity along the magnetic field, $\langle\ldots\rangle$ means bounce/transit time averaging, $l$ and $s$ are integers, $\dot{\varphi} \approx v_{\|} / R,\langle\dot{\varphi}\rangle=\omega_{D}$ for the trapped particles and $\langle\dot{\varphi}\rangle=\omega_{D}+\sigma_{v} q \omega_{b}$ with $\sigma_{v} \equiv \operatorname{sgn} v_{\|}$for the circulating ones with $q$ the safety factor, $\omega_{D}$

is the precession frequency, $\delta(x)$ is the Dirac $\delta$-function, and $\tilde{X}$ is proportional to the magnetic field perturbation. 
It follows from Eq. (1) that, in general, a particle interacts with the ripple through the following resonance condition:

$$
l\left\langle\omega_{B}\right\rangle-s \omega_{b}-N\langle\dot{\varphi}\rangle=0
$$

The resonance (4) is the large-time-scale one, i.e., it manifests itself for time intervals well exceeding the particle bounce/transit time. We will refer to it as the "global" resonance because it is determined by the particle motion along the whole bounce orbit. There exists a "local" resonance which manifests itself on a small part of the guiding center orbit. This resonance is well-known and can be obtained by assuming that the waveparticle interaction lasts for $\Delta t \ll \omega_{b}^{-1}$ (but $\Delta t \gg \omega_{B}^{-1}$ ):

$$
l \omega_{B}=N \dot{\varphi}
$$

The presence of a perturbation not necessarily leads to diffusion, i.e., particle orbits may remain regular. But if diffusion occurs (the conditions of the appearance of the ripple-induced diffusion are obtained in Sec. V), it is realized along the characteristics of the equation $\hat{\Pi} F=0$, i.e., along the straight lines in the $\{P, \mu\}$ space determined by

$$
P+\frac{N B}{l \omega_{B}} \mu=\text { const. }
$$

In the absence of the ripple, Eq. (6) yields an evident condition $P=$ const. Note that Eq. (6) can be obtained also by using the symmetry properties of the resonant term in the particle Lagrangian, see Sec. III. As shown in Sec. V, it represents an adiabatic invariant of the resonant motion.

According to the quasi-linear theory, the diffusion coefficient is proportional to $|\mathcal{H}|^{2}$, see Ref. ${ }^{4}$. This implies that $\mathcal{H}$ is essentially the normalized jump that a particle experiences during the random walk. The integral that determines $\mathcal{H}$ contains large parameters $(N$ and $\left.\omega_{B} / \omega_{b}\right)$ in the argument of the exponent in the integrand. Therefore, only the points determined by the local resonance condition mainly contribute to the integral. If the local resonance cannot occur during the particle orbital motion, $\mathcal{H}$ is exponentially small. Note that local resonances result in jumps of particles even without a global resonance, but then the effects of the jumps do not accumulate. 


\section{A. GWB diffusion ( $l=0$ resonance)}

Certain conclusions can be drawn by analyzing the fulfillment of the resonance conditions. When $l=0$, the global resonance is essentially the resonance between the particle poloidal and toroidal motions, whereas the local one determines the particle turning points, $\dot{\varphi}=0$. Both these resonances are responsible for the GWB diffusion. The ripple-particle interaction through these resonances does not change the particle magnetic moment (a term proportional to $\partial / \partial \mu$ is absent in Eq. (3) for $l=0$ ). Therefore, the radial diffusion occurs in the way that the banana tips ( $\dot{\varphi}=0$ points) lie on the curves of $B(R, Z)=$ const, where $Z$ is a coordinate in the vertical direction. In STs they may considerably differ from the $R=$ const lines, especially in the region where the ripple is large. They can even be closed, see Fig. 1. The particles with the banana tips lying on these curves will not diffuse from a plasma.

Let us consider the bounce and precession frequencies, which are contained in Eq. (4). Restricting ourselves to the narrow-orbit well-trapped particles, we can use the magnetic field in the form $B(\theta)=B(\theta=0)+B^{\prime \prime} \theta^{2} / 2$, where $B^{\prime \prime}=\partial^{2} B /\left.\partial \theta^{2}\right|_{\theta=0}$. Then we obtain $\omega_{b}=v \sqrt{\lambda B^{\prime \prime} /\left(2 B_{0}\right)} B_{p} /(r B)$ and $\omega_{D}=-\left(q v_{D 0} / r\right) k^{-1} B_{0}^{-1} \partial \ln B /\left.\partial \epsilon\right|_{\theta=0}$, where $B_{0}$ is the magnetic field at the magnetic axis, $B_{p}$ is the poloidal magnetic field, $v_{D 0}=\rho v /\left(2 R_{0}\right)$ is the characteristic drift velocity, $\rho=v / \omega_{B 0}, \omega_{B 0}=e B_{0} /(M c), k$ is the elongation of the plasma cross section, $r$ and $\theta$ are the radial (flux) and poloidal coordinates, respectively, and $\epsilon=r / R_{0}$. The magnitude of $\omega_{D} / \omega_{b}$ grows with the ratio of Larmor radius to the plasma size; therefore, in STs it can be much larger than in CTs. To evaluate $\omega_{D} / \omega_{b}$, at first, we take the magnetic field in the "standard" form, $B=B_{0}(1-\epsilon \cos \theta)$. Using NSTX parameters $\left(R_{0}=85 \div 100 \mathrm{~cm}, B=0.3 \mathrm{~T}, \mathcal{E}=80 \mathrm{keV}\right)$ and assuming $\epsilon=0.5, q=$ $\epsilon B / B_{p}=1.5$, we find that $\omega_{D} / \omega_{b} \sim 1 / 2$, which indicates that well-trapped particles may be characterized by $\omega_{D} / \omega_{b} \lesssim 1$. Furthermore, in high- $\beta$ discharges of STs $B^{\prime \prime}$ decreases, thus decreasing the bounce frequency ( $B^{\prime \prime}$ can even become negative, in which case teardrop orbits appear $\left.{ }^{8}\right)$. When $B^{\prime \prime}<\epsilon$ but $|\partial B / \partial r| \geq R_{0}^{-1}$ then $\omega_{D} / \omega_{b} \geq 1$. This implies that points with $v_{\|}=0$ disappear (although a particle can remain trapped in the poloidal 
direction), which reduces the number of particles affected by the GWB diffusion.

The calculations carried out with the code ORBIT ${ }^{5,6}$ confirmed these conclusions. Calculations were performed for $80-\mathrm{keV}$ deuterium ions in an NSTX plasma with $\beta=25 \%$. The considered equilibrium is characterized by the presence of a minimum of the magnetic field strength (the "magnetic valley") near the outer edge of the torus, see Fig. 1. Particles were launched from points with $\theta=0$. Trapped particles located in the outer region where $\partial B / \partial r>0$ were found, whereas in the internal region, where $\partial B / \partial r<0$, particles that were trapped poloidally but passing in the toroidal direction were observed. The former were characterized by $\omega_{D} / \omega_{b} \sim 1 / 3-1 / 5$.

Thus, in STs $\omega_{D} \lesssim \omega_{b}$. Therefore, large- $s$ resonances take place $(s \sim N \gg 1)$. A question arises of how it affects the GWB diffusion. To clarify this question, we approximate the particle motion along the large azimuth of the torus by

$$
\varphi=\omega_{D} t+q \theta_{b} \sin \omega_{b} t
$$

$\left(\theta_{b}>0\right.$ is the bounce angle), which is justified for the well trapped particles. Here the first term describes slow regular motion due to precession, whereas the second one describes bounce oscillations. If the condition of global resonance is satisfied, Eq. (7) takes the form:

$$
N \varphi=-s \tau+\xi \sin \tau
$$

where $\tau=t \omega_{b}, \xi=N q \theta_{b}$. It follows from Eq. (8) that the local resonance, $\dot{\varphi}=0$, occurs at the moments $\tau_{*}$ determined by $\cos \tau_{*}=s / \xi$. This implies that the local resonance is possible provided that the global one is characterized by $|s|<\xi$. Furthermore, the local resonance occurs near $\theta_{b}$ only for $|s| \ll \xi$; when $|s| \lesssim \xi$, it approaches the equatorial plane of the torus. Because of this, the stationary-phase method, which one usually uses to calculate the radial jumps caused by the ripple is applicable only when $|s|<\xi$. The mentioned method yields for $\mathcal{H}$ :

$$
\mathcal{H}=\omega_{b}^{-1} \int_{0}^{2 \pi} d \tau e^{i \phi}=\omega_{b}^{-1} \sum_{*=1,2} \sqrt{\frac{2 \pi}{\left|\ddot{\phi}_{*}\right|}} \exp \left\{i\left(\phi_{*}+\frac{\pi}{4} \operatorname{sgn} \ddot{\phi}_{*}\right)\right\}
$$


where $\phi=N \varphi,\left|\ddot{\phi}_{*}\right| \equiv N\left|d^{2} \varphi / d \tau^{2}\right|=\sqrt{\xi^{2}-s^{2}}$. We observe that Eq. (9) predicts $\mathcal{H} \rightarrow \infty$ for $|s| \rightarrow \xi$ (i.e., infinite radial jumps of the particle). On the other hand, the integral in Eq. (2) can be easily calculated exactly:

$$
\mathcal{H}=\tau_{b} J_{s}(\xi)
$$

where $J_{s}(\xi)$ is the Bessel function, which can be approximated as follows:

$$
J_{s}(\xi) \approx \sqrt{\frac{2}{\pi \xi}} \cos \left(\xi-\frac{\pi s}{2}-\frac{\pi}{4}\right) \text { for } s \ll \xi, \quad J_{s}(\xi) \approx \frac{0.45}{\xi^{1 / 3}} \text { for } s=\xi>1 .
$$

Note that the amplitude in the first expression of Eq. (11) exceeds $J_{s}(s)$ unless $\xi>31$. We conclude from here that the stationary-phase method provides a good approximation when $s \ll \xi$ (then $\ddot{\phi}_{*} \approx \xi$ ). The same formula for $\mathcal{H}$ or a more appropriate one given by the second expression of Eq. (11) can be used for $|s| \lesssim \xi$. When $|s| \gg \xi, \mathcal{H}$ is very small and rapidly decreases with $s$.

\section{B. CRI diffusion $(l \neq 0$ resonance $)$}

Using Eqs. (6) and (4), we find that the change of the magnetic moment, $\Delta \mu$, and the change of the canonical angular momentum, $\Delta P$, during the diffusion are connected as follows:

$$
\Delta P=-\frac{\langle B\rangle \Delta \mu}{\langle\dot{\varphi}\rangle+s \omega_{b} / N}
$$

In general, this equation implies that when $\Delta \mu \neq 0$, the particles move across the flux surfaces. But this does not necessarily mean that the particles will escape from the plasma.

Let us consider well-circulating particles. For them, using the definition of $P$, we obtain the relationship

$$
\Delta P \approx-\frac{e}{c} \Delta\langle\psi\rangle-\frac{B_{0} R_{0}}{v_{\|}} \Delta \mu
$$

Combining Eqs. (12) and (13) and using $\Delta \psi=B_{0} k r \Delta r / q$, we find: 


$$
\Delta\langle r\rangle \approx-\frac{q \rho}{2 k \epsilon} \frac{\sigma_{v} \omega_{D} / \omega_{b}+s / N}{q+\sigma_{v} \omega_{D} / \omega_{b}+s / N} \Delta \lambda
$$

where $\lambda=\mu B_{0} / \mathcal{E}$ with $\mathcal{E}=M v^{2} / 2$ is the pitch-angle parameter. In this equation, $s$ is zero or not more than of the order of unity; otherwise, the conditions of the local and global resonances are not consistent. Therefore, we neglect the sideband resonances. Then, if the toroidal precession is negligible, which is the case in CTs, Eq. (14) yields $\Delta\langle r\rangle \approx 0$. But in high- $\beta$ plasmas of STs the precession of well-circulating particles may be rather strong because of the large Shafranov shift. ${ }^{7}$ Taking $\omega_{D}=\hat{\xi} \rho v / R_{0}^{2}$, where $\hat{\xi}>1$ is a parameter determined by the Shafranov shift, ${ }^{7}$ we obtain:

$$
\frac{\Delta\langle r\rangle}{\langle r\rangle} \approx-\frac{\sigma_{v} \hat{\xi} q \Delta \lambda}{2 k\left(1+\sigma_{v} \hat{\xi} \rho / R_{0}\right)} \frac{\rho^{2}}{\langle r\rangle^{2}},
$$

For instance, for $q=1.5, k=1.5, \rho / R_{0}=0.2, \xi=3$, and $\Delta \lambda=0.5$, Eq. (15) yields $\Delta\langle r\rangle /\langle r\rangle=-0.5\left(\rho^{2} /\langle r\rangle^{2}\right.$. Thus, the diffusion of the well-circulating particles, even when precession is strong, can hardly result in a considerable particle displacement.

However, trapped and marginally circulating particles may be strongly influenced. This conclusion results from the following equation obtained from $P \approx-e\langle\psi\rangle / c$ and Eq. (6):

$$
\frac{\Delta\langle r\rangle}{\langle r\rangle} \approx \frac{N q \Delta \lambda}{2 k l} \frac{\rho^{2}}{\langle r\rangle^{2}}
$$

Equation (16) shows that $\Delta r$ can be about the plasma radius due to the relatively large ratio $\rho^{2} / a^{2}$ in STs. But this will be the case only when the resonance width is sufficient for the resonances to overlap in a large part of the plasma.

It is of importance to know the trajectories of the motion of the resonance points during the diffusion. An equation for them can be obtained by using Eqs. (5), (6), and the definitions of $P$ and $\mu$. We find:

$$
\frac{2 l}{N} \psi-\frac{l^{2} R^{2} B}{N^{2}}-\rho^{2} B=\mathrm{const}
$$

where $\psi=\psi(R, Z), \rho=\rho(R, Z), B=B(R, Z)$. The second term here is typically small. Taking this into account, we conclude that when $\rho$ is small, the resonance points 
move approximately along flux surfaces; therefore, the motion does not lead to a loss of the particles (unless the particles strike the wall because of finite $\rho$ during this motion). On the contrary, when $\rho$ is large, the resonance points move approximately along the $B=$ const lines (as in the case of the GWB diffusion), which may result in escape of the particles to the wall. To obtain corresponding conditions, it is convenient to eliminate $l$ in Eq. (17) by means of Eq. (5):

$$
2 \chi \frac{\rho}{R} \psi-B \rho^{2}\left(1+\chi^{2}\right)=\text { const }
$$

where $\chi(R, Z) \equiv v_{\|} / v=l R /(N \rho)$ is the pitch angle in the point of the local resonance. Note that $\chi=$ const when $B R=$ const. It follows from Eq. (18) that the particles move approximately along the flux surfaces and, therefore, are well-confined when (we assume that the change of $B$ during the motion is $\sim \epsilon B_{0}$ )

$$
\frac{\rho}{r} \ll \frac{k \chi}{q\left(1+\chi^{2}\right)}
$$

Equation (19) can be easily satisfied in CTs, but not in STs. For instance, Eq. (19) is not satisfied for $80-\mathrm{keV}$ deuterons in NSTX with $B \approx 0.3 \mathrm{~T}$. These energetic deuterons interact with the ripple through $l= \pm 1, \pm 2$, and \pm 3 resonances at $\chi \approx \pm 0.3, \pm 0.6$, and \pm 0.9 , respectively. One can see that most trajectories for the $l= \pm 1$ resonance cross the wall when $B R=$ const (in which case the orbits of the resonance points are the ellipses shifted with respect to the flux surfaces). But on the other hand, in high- $\beta$ discharges particle can be confined even when resonance points move along the $B=$ const contours provided that these contours are closed, see Fig. 1 (as we already mentioned considering the GWB diffusion).

To evaluate $\mathcal{H}$, we take $B=B_{0}(1-\epsilon \cos \theta)$ and consider again well trapped particles described by Eq. (8). We then obtain:

$$
\mathcal{H}=\sum_{p} J_{p}(\eta) J_{s+2 p}(\xi)
$$

where $\eta \equiv l \omega_{B} \epsilon \theta_{b}^{2} /\left(8 \omega_{b}\right)$. When $l \sim 1$, typically $\eta<1$; therefore, $\mathcal{H} \approx J_{s}(\xi)$, as in the $l=0$ case. This is explained by the fact that the point $\tau_{*}$ satisfies an equation which 
approximately coincides with the one for the $l=0$ diffusion when $\eta \ll \xi$. This can be seen from the equation $\phi=-s \tau+\xi \sin \tau-\eta \sin (2 \tau)$. However, different values of $s$ are relevant to the GWB diffusion and CRI diffusion because the points determined by Eq. (5) with $l=0$ and $l \neq 0$ are different. For instance, $|s|=| \pm 15-4|$ for $l= \pm 1$ and $|s|=4$ for $l=0$ when $\omega_{b} /\left|\omega_{D}\right|=4, \omega_{B} / \omega_{b}= \pm 15, N=16$. We observe that the cyclotron resonance is characterized by higher magnitudes of $|s|$ with $|s|_{\min }<\xi\left(|s|_{\min }=9, \xi=16 q \theta_{b}\right)$. Particles in this resonance experience a jump slightly exceeding the $l=0$ jump.

\section{Garren effect (imaginary local resonance)}

It was shown by Garren et $a .^{3}$ that the particle magnetic moment $(\mu)$ oscillates about a fixed value with the gyrofrequency until the particle crosses the median plane of the mirror machine, near which $\mu$ suffers a relatively large transient change $(\delta \mu)$. In general, this means that a particle experiences small kicks when crossing minima of the magnetic field. The change of the magnetic moment, $\delta \mu$, found in Ref. ${ }^{3}$ scales with the particle velocity and the magnetic field as $\exp \left\{-c_{1} B / v\right\}$ with $c_{1}=$ const, which implies that the effect may be of importance for fast particles moving in a weak magnetic field. Later a formula for $\delta \mu$ was suggested..$^{9-11}$ Below we use a little bit different formula derived in Sec. III (where, in addition, a comparison of our result with the previously obtained one is carried out):

$$
\delta \lambda=2 v \sqrt{\lambda} \int_{t_{1}}^{t_{2}} d t\left(\frac{B_{0}}{B}\right)^{1 / 2} \frac{1}{R_{c}}\left(1-\frac{\lambda B}{B_{0}}\right) \cos (\Phi)
$$

where $\Phi=\Phi_{0}-\int_{0}^{t} d t^{\prime} \omega_{B}$ is the gyrophase, $t_{1}$ and $t_{2}$ are the turning points, $R_{c}$ is the radius of the field line curvature. We observe that $\delta \lambda$ given by Eq. (21) is proportional to an integral similar to $\mathcal{H}$ with $N=0$ except for the presence of an additional weight function. Therefore, Garren's kicks are essentially sharp changes of the drift invariants in the absence of a local resonance. But on the other hand, below we will show that the point in the complex plane $t_{*} \equiv \operatorname{Re} t_{*}+i \operatorname{Im} t_{*}$ where $B\left(t_{*}\right)=0$ mainly contributes to the integral in Eq. (21) for the particles satisfying Eq. (4) with $N=0, l=1$. For 
this reason, one can say that Garren's kicks are caused by an imaginary local resonance determined by the resonance condition given by Eq. (5) with $N=0$ and $l \neq 0$. We can easily calculate $\mathcal{H}$ exactly (without the use of the steepest-descent method, which one usually applies to calculate $\delta \mu$ ), restricting ourselves to the case of either well-circulating or well-trapped particles. To make calculations for circulating particles, we replace the integral $\int_{t_{1}}^{t_{2}} d t$ in Eq. (21) by $\oint d t$, which corresponds to Eq. (2). We consider the particles satisfying the global resonance condition, for which the displacements accumulate, and take the magnetic field in the form $B=B_{0}(1-\epsilon \cos \theta)$. Then, taking $\theta=\tau$ with $\tau=t \omega_{b}$ for the circulating particles and $\theta=\theta_{b} \sin \tau$ for the trapped ones, we obtain:

$$
\mathcal{H}^{c}=\tau_{b} J_{s}(s \epsilon), \quad \mathcal{H}^{t}=\tau_{b} J_{n}\left(n \epsilon \theta_{b}^{2} / 4\right)
$$

where $|s|=\left\langle\omega_{B}\right\rangle / \omega_{b}$ and $n=s / 2$ are integers, the superscripts $t$ and $c$ are relevant to trapped and circulating particles, respectively. Because $s \gg 1$ and $\epsilon \ll 1$, Eq. (22) can be approximated as follows:

$$
\mathcal{H}^{c} \approx \frac{\tau_{b}^{c}}{\sqrt{2 \pi|s|}} \exp \left\{-|s|\left(\ln \frac{2}{\epsilon}-1\right)\right\}, \quad \mathcal{H}^{t} \approx \frac{\tau_{b}^{t}}{\sqrt{2 \pi|n|}} \exp \left\{-|n|\left(\ln \frac{8}{\epsilon \theta_{b}^{2}}-1\right)\right\} .
$$

Equation (23) provides a good approximation only for sufficiently small $\epsilon$; otherwise, it overestimates the Bessel functions. To find the point that mainly contributes to the integral, we recall that according to the steepest-descent method (which is applicable due to the large ratio $\left.\left\langle\omega_{B}\right\rangle / \omega_{b}\right), \tau$ should be considered as a complex variable and that the saddle point of the phase is determined by the equation $\dot{\Phi}=0$, i.e., $B\left(\tau_{*}\right)=0$. The main contribution to the integral for the resonant particles comes from the vicinity of this point (for these particles, the contribution of the end points vanishes as $\Phi(\tau+2 \pi)=\Phi(\tau)+2 \pi s$ ). In the considered case $\operatorname{Re} \tau_{*}=0$, which means that $\theta_{*}=0$. Thus, as expected, the point where $B(\theta)$ has a minimum is responsible for the jump of $\mu$. It follows from Eq. (23) that $\mathcal{H}$ exponentially decreases with the resonance number $(s)$. On the other hand, $\mathcal{H}$ strongly depends on $\epsilon$, which is essentially $B^{\prime \prime}\left(\theta=\theta_{*}\right)$.

In order to evaluate $\delta \lambda$, we approximate the radius of the field line curvature as $R_{c}=R_{0}(1+\epsilon \cos \theta)$, see Appendix $\mathrm{A}$, in which case $B R_{c} \approx$ const. Then, taking into account that the point $\tau_{*}$ mainly contributes to the integral, we obtain (we take $\Phi_{0}=0$ ): 


$$
\delta \lambda=\frac{\sqrt{2 \lambda} v}{R_{0}} \mathcal{H} .
$$

Note that Eq. (24) is obtained from Eq. (21), which includes effects of the plasma pressure. However, these effects were disregarded when we took $B R_{c}=$ const. On the other hand, the influence of plasma pressure on $R_{c}$ is rather small (see Appendix A) although the field line curvature is $\kappa=\kappa_{1}+\kappa_{2}$, where $\kappa_{1} \propto \nabla_{\perp} B, \kappa_{1} \propto \nabla_{\perp} p$. When $\beta$ becomes high, $\left|\kappa_{2}\right|$ becomes considerable, but its increase is compensated for by a corresponding change of $\kappa_{1}$. Taking $R_{c}=R_{0}(1+\epsilon \cos \theta)$, we avoid the risk to overestimate considerably $\delta \lambda$ by calculating separately the integrals associated with $\kappa_{1}$ and $\kappa_{2}$, as would be done if we used, e.g., the general formula for $\delta \lambda$ presented in Ref. ${ }^{11}$. [The integrals in Eq. (21) may be rather sensitive to the truncation of terms of high order in $\epsilon \cos \theta$, which can be seen from, e.g., the fact that $\oint d t \omega_{B}(t) \exp (i \Phi)=i \oint d \exp (i \Phi)=0$ for the particles satisfying the condition $\left\langle\omega_{B}\right\rangle=s \omega_{b}$, where $s$ is an arbitrary integer.] Thus, one can think that Eq. (24) represents a reasonable estimate for the $\lambda$ jumps in low- $\beta$ plasmas.

In discharges with relatively high $\beta$ (e.g., in current experiments on NSTX with $\beta$ not exceeding $25 \%) B(\theta=0)=B_{\min }$ but $\partial B / \partial r>0$ at $\theta=0$ in some region outwards the magnetic axis. The magnetic field in this case can be approximated as $B=B_{0}(1-$ $\left.\epsilon \cos \theta+\alpha \epsilon^{2}\right)$ with $2 \alpha \epsilon>1$ in the region of the magnetic valley. One can show that in this case the factor $\ln (2 / \epsilon)$ in Eq. (23) must be replaced by $\ln \left(2 \epsilon^{-1}+\alpha \epsilon\right)$. This implies that the magnetic valley (the region with $\partial B / \partial r>0$ at $\theta=0$ ) reduces $\delta \mu$. The obtained result has a simple explanation: in the presence of the valley the lines of $B=$ const deflect from the $R=$ const lines in the region where $B$ is minimum in such a way that the variation of $B$ during a passage of a particle through the minimum-B region is reduced, see Fig. 1 .

In plasmas with very high $\beta, B(\theta)$ may have a maximum at $\theta=0$. Then jumps of $\mu$ at $\theta=0$ disappear; one can expect that instead of them small jumps will occur above and below the midplane of the torus.

When $\delta \lambda($ or $\delta \mu)$ is sufficiently small, the particle motion remains regular, i.e., "superadiabatic." 12,13 In the contrary case, when $\delta \lambda$ is sufficiently large, stochasticity arises. However, its effect on the bounce-averaged orbits of trapped particles will be 
negligible: for these particles $P \approx-(e / c)\langle\psi\rangle$; therefore, $\delta\langle\psi\rangle \approx 0$. A jump of $\lambda$ of a circulating particle results in a radial jump according to the following equation (we use $P=\sigma_{v} M q R_{0}^{2} \omega_{b}-(e / c)\langle\psi\rangle=$ const $):$

$$
\delta\langle r\rangle=-\frac{\sigma_{v} q \rho(1-\epsilon)}{2 \epsilon \chi_{m} k} \delta \lambda
$$

where $\chi_{m} \equiv \sqrt{1-\lambda(1-\epsilon)}$ is the particle pitch angle at $\theta=0$, and

$$
\delta \lambda=\frac{4 \pi q \sqrt{\lambda}}{\sqrt{2} \chi_{m}} J_{\nu}(s \epsilon) .
$$

We observe that $\delta \lambda$ and $\delta\langle r\rangle$ vanishes for the "most circulating" particles, i.e., for the particles with $\lambda=0$ (but $\delta \mu / \mu=\delta \lambda / \lambda=\infty$ for them!). A discussion of a possible role of the stochastic diffusion is contained in Sec. VI.

\section{LAGRANGIAN FORMALISM FOR THE DESCIPTION OF THE NONADIABATIC EFFECTS IN THE PARTICLE MOTION}

In our consideration of the particle motion, we follow a perturbative approach. We approximate the exact Lagrangian of the particle motion in the magnetic field of a spherical torus by the Lagrangian of a certain integrable system and consider the difference between the exact and integrable Lagrangians as a perturbation. For such an approach to be justified, the integrable Lagrangian (which we consider as describing the motion of the unperturbed system) should be chosen in such a way that the perturbation defined so is small.

We split the magnetic field into the axisymmetric part, $\mathbf{B}$, and the small nonaxisymmetric component (ripple), $\tilde{\mathbf{B}}$. Then we choose the lowest-order Lagrangian of the guiding-center motion ${ }^{14}$ in the axisymmetric field $\mathbf{B}$,

$$
\mathcal{L}_{g c}(\mathbf{X}, \mathcal{E}, \mu, \dot{\mathbf{X}}, \dot{\Phi})=\frac{e}{c} \mathbf{A}(\mathbf{X}) \cdot \dot{\mathbf{X}}+\frac{M v_{\|}}{B} \mathbf{B}(\mathbf{X}) \cdot \dot{\mathbf{X}}+\frac{M c}{e} \mu \dot{\Phi}-\mathcal{E}
$$

as the unperturbed system (we neglect the effect of the equilibrium electric field on the particle motion). Here $\mathbf{X}$ is the guiding center position; $e, M$, and $\mathcal{E}$ are the charge, mass, and energy of the particle; $\Phi$ is the gyrophase; $\mathbf{A}$ and $\tilde{\mathbf{A}}$ are the vector potentials 
corresponding to $\mathbf{B}$ and $\tilde{\mathbf{B}}$, respectively; $v_{\|}=\{2[\mathcal{E}-\mu B(\mathbf{X})] / M\}^{1 / 2}$ is considered as a function of $\mathbf{X}, \mathcal{E}$ and $\mu$. The guiding center position, $\mathbf{X}$, and the particle position, $\mathbf{x}$, are connected to the considered order by the relation $\mathbf{x}=\mathbf{X}+v_{\perp} \hat{\mathbf{a}} / \omega_{B}$, where $\hat{\mathbf{a}}=$ $\cos (\Phi) \hat{\mathbf{e}}_{1}(\mathbf{X})-\sin (\Phi) \hat{\mathbf{e}}_{2}(\mathbf{X}), \hat{\mathbf{e}}_{1}$ and $\hat{\mathbf{e}}_{2}$ are arbitrary unit vectors constituting with the vector $\hat{\mathbf{b}}=\mathbf{B} / B$ a local orthonormal base and slowly varying in space (we take the normal to the magnetic field line as $\hat{\mathbf{e}}_{2}$ and the binormal, as $\left.-\hat{\mathbf{e}}_{1}\right)$. The Lagrangian given by Eq. (27) describes an integrable system, possessing three constants of motion: $\mathcal{E}, P$, and $\mu$.

The exact Lagrangian of a particle in the rippled magnetic field is

$$
\mathcal{L}(\mathbf{x}, \mathbf{v}, \dot{\mathbf{x}})=\frac{e}{c}[\mathbf{A}(\mathbf{x})+\tilde{\mathbf{A}}(\mathbf{x})] \cdot \dot{\mathbf{x}}+M \mathbf{v} \cdot \dot{\mathbf{x}}-\frac{M}{2} v^{2},
$$

Note that this Lagrangian, as well as that given by Eq. (27), differs from the Lagrangians that one usually meets in analytical mechanics. First of all, it depends on six (rather than three) phase space variables, $\mathbf{x}$ and $\mathbf{v}$, and their time derivatives. During the variational process, the variations of all the phase space variables are to be considered independent even though the equations of motion imposes some functional dependence on them. In particular, $\mathbf{v}$ and $\dot{\mathbf{x}}$ in Eq. (28) are considered as independent variables. The larger number of variables is compensated for by the fact that the Lagrangian depends on them linearly. This property facilitates using the Lie transform methods to construct perturbation schemes (a more detailed discussion of related topics can be found in Refs. ${ }^{14,15}$ ).

Equation (28) can be presented as follows:

$$
\mathcal{L}=\mathcal{L}_{g c}+\underbrace{\left(\mathcal{L}_{a s}-\mathcal{L}_{g c}\right)}_{\delta \mathcal{L}_{1}}+\underbrace{\left(\mathcal{L}-\mathcal{L}_{a s}\right)}_{\delta \mathcal{L}_{2}}=\mathcal{L}_{g c}+\delta \mathcal{L}_{1}+\delta \mathcal{L}_{2},
$$

where

$$
\mathcal{L}_{a s}(\mathbf{x}, \mathbf{v})=\frac{e}{c} \mathbf{A}(\mathbf{x}) \cdot \dot{\mathbf{x}}+M \mathbf{v} \cdot \dot{\mathbf{x}}-\frac{M}{2} v^{2}
$$

is the Lagrangian of the particle motion in the axisymmetric field. We will consider $\delta \mathcal{L}_{1}$ and $\delta \mathcal{L}_{2}$ as perturbations. The first of them,

$$
\delta \mathcal{L}_{1}=\frac{e}{c} \mathbf{A}(\mathbf{x}) \cdot \dot{\mathbf{x}}+M \mathbf{v} \cdot \dot{\mathbf{x}}-\frac{e}{c} \mathbf{A}(\mathbf{X}) \cdot \dot{\mathbf{X}}-\frac{M v_{\|}}{B} \mathbf{B}(\mathbf{X}) \cdot \dot{\mathbf{X}}-\frac{M c}{e} \mu \dot{\Phi}
$$


describes the difference between the exact and guiding-center descriptions of the particle motion and is responsible for all nonadiabatic effects in the axisymmetric field (including the Garren effect). The second one,

$$
\delta \mathcal{L}_{2}=\frac{e}{c} \tilde{\mathbf{A}}(\mathbf{x}) \cdot \dot{\mathbf{x}}
$$

represents the effect of the ripple. Note that we avoid using the guiding-center approximation for the particle motion in the rippled field because the characteristic length of the ripple variation in, e.g., NSTX is not large in comparison with the Larmor radius of fast ions (80-keV deuterons). On the other hand, the characteristic variation length of the axisymmetric part of the field strength is large in comparison with the Larmor radius of the particles under consideration. This gives us grounds to assume that the error of the guiding-center approximation in the axisymmetric field, which is given by $\delta \mathcal{L}_{1}$, is small and, thus, that the perturbative approach is justified (the equations for the variation of $\mu$ given below can be used to determine more exactly when this assumption is true).

As the Lagrangian system given by Eq. (27) is integrable, each trajectory of the system lies on a certain three-dimensional torus in the phase space (a Kolmogorov-ArnoldMoser, or KAM, torus), ${ }^{16}$ which is in our case determined by the equations $\mathcal{E}=$ const, $\mu=$ const, $P=$ const. The position of a particle on the torus can be described by three angular coordinates, which can be chosen so that they depend linearly on time along the trajectories (so-called canonical angles): ${ }^{16}$

$$
\dot{\varsigma}_{1}=\left\langle\omega_{B}\right\rangle(\mathcal{E}, \mu, P), \quad \dot{\varsigma}_{2}=\omega_{b}(\mathcal{E}, \mu, P), \quad \dot{\varsigma_{3}}=\omega_{\varphi}(\mathcal{E}, \mu, P)
$$

where $\varsigma_{i}, i=1,2,3$, are the canonical angles describing the gyrophase, the poloidal position and the toroidal position, respectively; $\omega_{\varphi}$ is the frequency of the toroidal motion. The canonical action-angle coordinates for the particle in an axisymmetric magnetic trap were first constructed in Ref. ${ }^{17}$ via the generating function of an appropriate canonical transformation. An easier way to find the connection between conventional and canonical coordinates is to use the constancy of the time derivatives of the canonical angles. We find: ${ }^{18}$ 


$$
\varsigma_{1}=\Phi-\varpi\left(\varsigma_{2}\right), \quad \varsigma_{2}=\omega_{b} \int_{0}^{\theta} d \theta / \dot{\theta}, \quad \varsigma_{3}=\varphi-v\left(\varsigma_{2}\right),
$$

where $v \equiv \int_{0}^{\varsigma_{2}} d \varsigma_{2}\left(\dot{\varphi}-\omega_{\varphi}\right) / \omega_{b}$ and $\varpi \equiv \int_{0}^{\varsigma_{2}} d \varsigma_{2}\left(\omega_{B}-\left\langle\omega_{B}\right\rangle\right) / \omega_{b}$ are continuous periodic functions of $\theta$; the integrals are taken along the guiding-center orbit $P(r, \theta, \mathcal{E}, \mu)=$ const, $\mu=$ const, $\mathcal{E}=$ const; $\dot{\theta}(\theta, P, \mathcal{E}, \mu)=\dot{\mathbf{X}} \cdot \nabla \theta$ and $\dot{\varphi}(\theta, P, \mathcal{E}, \mu)=\dot{\mathbf{X}} \cdot \nabla \varphi($ when $\epsilon / q \ll 1$, $\left.\dot{\varphi} \approx v_{\|} / R\right)$ are angular velocities of the unperturbed guiding-center motion.

On transforming to the canonical angles, the Lagrangian $\mathcal{L}_{g c}$ can be written in the form (cf. Ref. ${ }^{18}$ )

$$
\mathcal{L}_{g c}=J_{1} \dot{\varsigma}_{1}+J_{2} \dot{\varsigma}_{2}+J_{3} \dot{\varsigma_{3}}-\mathcal{E}
$$

where $J_{1}=\mu M c / e, J_{3}=P$, and

$$
J_{2}\left(J_{1}, J_{3}, \mathcal{E}\right)=\frac{1}{2 \pi} \oint d \mathbf{X} \cdot\left[\frac{e}{c} \mathbf{A}(\mathbf{X})+\frac{M v_{\|}}{B} \mathbf{B}(\mathbf{X})\right]
$$

are the action variables canonically conjugate to $\varsigma_{1}, \varsigma_{2}$, and $\varsigma_{3}$, respectively; the integral in this equation is taken along a contour that is determined by the equations $P(r, \theta, \mu, \mathcal{E})=$ const, $\mu=$ const, $\mathcal{E}=$ const (i.e., lies on the corresponding invariant torus of the system) and makes one turn in the poloidal direction and no turns in the toroidal direction. Note that for narrow banana orbits $J_{2}$ becomes the longitudinal adiabatic invariant, $\oint d s m v_{\|} /(2 \pi)$, where the integral is taken along a field line between the orbit bounce points, $d s$ is the differential of the field line length. If we considered $\mathcal{E}$ as a function of $J_{1}, J_{2}$ and $J_{3}$ (the Hamiltonian of the system), the Euler-Lagrange equations following from Eq. (35) would be conventional Hamilton's equations in action-angle coordinates. However, it is more practical to consider $J_{2}$ as a function of $J_{1}, J_{3}$, and $\mathcal{E}$. Thus, we use $\varsigma_{1}, \varsigma_{2}, \varsigma_{3}, J_{1}, J_{3}$, and $\mathcal{E}$ as coordinates. Then the Euler-Lagrange equations are as follows:

$$
\begin{gathered}
\dot{J}_{1}=\dot{J}_{2}=\dot{J}_{3}=0 \\
\dot{\varsigma}_{1} \equiv \omega_{1}=-\left(\frac{\partial J_{2}}{\partial \mathcal{E}}\right)^{-1} \frac{\partial J_{2}}{\partial J_{1}}=\left\langle\omega_{B}\right\rangle \\
\dot{\varsigma}_{2} \equiv \omega_{2}=\left(\frac{\partial J_{2}}{\partial \mathcal{E}}\right)^{-1}=\omega_{b}
\end{gathered}
$$




$$
\dot{\varsigma}_{3} \equiv \omega_{3}=-\left(\frac{\partial J_{2}}{\partial \mathcal{E}}\right)^{-1} \frac{\partial J_{2}}{\partial J_{3}}=\omega_{\varphi}
$$

\section{DERIVATION OF A FORMULA FOR GARREN KICKS}

We proceed from Eq. (31), which gives the difference between the exact and guidingcenter descriptions of the particle motion in the axisymmetric field. Assuming that the field variation scale is much larger than the gyroradius, we expand $\mathbf{A}(\mathbf{x})$ in a power series in $\mathbf{x}-\mathbf{X}=v_{\perp} \hat{\mathbf{a}} / \omega_{B}(\mathbf{X})$. The resulting rather cumbersome expression for $\delta \mathcal{L}_{1}$ is considerably simplified on using the following property of the Lagrangian formalism: The equations of motion are invariant under the substitution $\mathcal{L} \rightarrow \mathcal{L}+d S / d t$, where $\mathrm{S}$ is an arbitrary function of $t$ and phase variables. In particular, it is known ${ }^{14}$ that the derivation of the guiding-center Lagrangian $\mathcal{L}_{g c}$ has included such a substitution with

$$
S=-\frac{e v_{\perp}}{c \omega_{B}(\mathbf{X})} \mathbf{A}(\mathbf{X}) \cdot \hat{\mathbf{a}}-\frac{e v_{\perp}^{2}}{c \omega_{B}(\mathbf{X})^{2}}(\hat{\mathbf{a}} \cdot \nabla) \mathbf{A}(\mathbf{X}) \cdot \hat{\mathbf{a}} .
$$

On applying this substitution to $\delta \mathcal{L}_{1}$, most terms are canceled, and we present $\delta \mathcal{L}_{1}$ to the leading order as follows:

$$
\delta \mathcal{L}_{1}=\frac{M v_{\|} v_{\perp}}{\omega_{B}(\mathbf{X})} \hat{\mathbf{b}} \cdot \xi+\frac{M v_{\perp}^{2}}{2 \omega_{B}(\mathbf{X})} \hat{\mathbf{e}}_{1} \cdot \frac{d \hat{\mathbf{e}}_{2}}{d t}+\frac{M^{2} c v_{\perp}^{2}}{2 e B(\mathbf{X})^{2}}(\hat{\mathbf{a}} \cdot \nabla) \mathbf{B}(\mathbf{X}) \cdot(\hat{\mathbf{a}} \times \dot{\mathbf{X}})
$$

where $\xi=\cos (\Phi) d \hat{\mathbf{e}}_{1} / d t-\sin (\Phi) d \hat{\mathbf{e}}_{2} / d t$.

To derive an equation for $\dot{\mu}$ in the axisymmetric field, we set $\delta \mathcal{L}_{2}=0$ and write the following Euler-Lagrange equation:

$$
\frac{d}{d t}\left(\frac{\partial \mathcal{L}_{g c}}{\partial \dot{\Phi}}+\frac{\partial \delta \mathcal{L}_{1}}{\partial \dot{\Phi}}\right)=\frac{\partial \delta \mathcal{L}_{1}}{\partial \Phi}
$$

from which it immediately follows that

$$
\dot{\mu}=\frac{e}{M c}\left[\frac{\partial \delta \mathcal{L}_{1}}{\partial \Phi}-\frac{d}{d t}\left(\frac{\partial \delta \mathcal{L}_{1}}{\partial \dot{\Phi}}\right)\right]
$$

Substituting $\delta \mathcal{L}_{1}$ from Eq. (42) and keeping only terms of the lowest order in the gyroradius, after straightforward calculations we obtain the following equation: 


$$
\dot{\mu}=\frac{M v_{\|} v_{\perp}}{B(\mathbf{X})} \hat{\mathbf{b}} \cdot \frac{\partial \xi}{\partial \Phi}+\frac{M v_{\perp}^{2}}{2 B(\mathbf{X})^{2}} \frac{\partial}{\partial \Phi}[(\hat{\mathbf{a}} \cdot \nabla) \mathbf{B}(\mathbf{X}) \cdot(\hat{\mathbf{a}} \times \dot{\mathbf{X}})] .
$$

The second term of the right-hand side of this equation is proportional to $\exp (2 i \Phi)$. Therefore, it gives an exponentially small contribution to jumps of the magnetic moment ${ }^{9}$ and will be ignored. Keeping only the longitudinal component of $\dot{\mathbf{X}}$ (the transversal component is of higher order in the gyroradius) and using the Frenet equations, we obtain:

$$
\begin{gathered}
\frac{d \hat{\mathbf{e}}_{1}}{d t} \approx v_{\|} \frac{d \hat{\mathbf{e}}_{1}}{d l}=-v_{\|} \tau \hat{\mathbf{e}}_{2}, \\
\frac{d \hat{\mathbf{e}}_{2}}{d t} \approx v_{\|} \frac{d \hat{\mathbf{e}}_{2}}{d l}=-v_{\|} \kappa \hat{\mathbf{b}}+v_{\|} \tau \hat{\mathbf{e}}_{1},
\end{gathered}
$$

where $d l$ is the the differential of the field line length, $\tau$ is the field line torsion. Substituting these equations to Eq. (45), we finally arrive at the following equation:

$$
\dot{\mu}=M \frac{v_{\|}^{2} v_{\perp}}{B R_{c}} \cos \Phi=M \frac{v^{2} v_{\perp}}{B R_{c}}\left(1-\frac{\lambda B}{B_{0}}\right) \cos \Phi,
$$

where we again restricted ourselves to the leading order in the gyroradius and omitted terms containing $\exp (2 i \Phi)$. Equation (48) is essentially Eq. (21).

Equation (48) seems to disagree with previous results of Refs. ${ }^{9,10}$. According to these works, the second term in the parentheses in Eq. (48) should be twice less. However, when the plasma pressure is negligible, the discrepancy is easily explained by the difference in the definitions of $\mu$ in Refs. ${ }^{9,10}$ and here. Here $\mu$ is defined in terms of the magnetic field at the guiding center, $\mu=M v_{\perp}^{2} /[2 B(\mathbf{X})]$, whereas in the mentioned previous works the magnetic field is taken at the particle position. In the latter case the particle gyration introduces additional terms into $\dot{\mu}$ :

$$
\begin{aligned}
\dot{\mu}_{l o c a l} & \equiv \frac{d}{d t}\left(\frac{M v_{\perp}^{2}}{2 B(\mathbf{x})}\right)=\dot{\mu}-\frac{\mu v_{\perp}}{B(\mathbf{X})} \hat{\mathbf{c}} \cdot \nabla_{\perp} B \\
& =\dot{\mu}+M \frac{v_{\perp}^{3}}{2 B R_{c}} \cos \Phi-M \frac{2 \pi v_{\perp}^{3}}{B^{3}}\left[\left(\hat{\mathbf{e}}_{2} \cdot \nabla p\right) \cos \Phi+\left(\hat{\mathbf{e}}_{1} \cdot \nabla p\right) \sin \Phi\right]
\end{aligned}
$$

where $\hat{\mathbf{c}}=\partial \hat{\mathbf{a}} / \partial \Phi=\hat{\mathbf{a}} \times \hat{\mathbf{b}}=-\sin (\Phi) \hat{\mathbf{e}}_{1}(\mathbf{X})-\cos (\Phi) \hat{\mathbf{e}}_{2}(\mathbf{X})$ is the instant direction of the particle gyromotion. The first two terms of Eq. (49) yield the equation for $\delta \mu$ of Refs. ${ }^{9,10}$. Therefore, we conclude that Eqs. (48) and (21) differ from the mentioned previous formulae by taking into account effects of plasma pressure described by last two terms of 
Eq. (49). Introducing a curvature-like parameter $8 \pi B^{-2} \nabla p$ in addition to $\kappa=R_{c}^{-1}$, we could obtain a formula for $\dot{\mu}_{\text {local }}$ similar to that derived in Ref. ${ }^{11}$. However, the equation for $\dot{\mu}$ given by Eq. (48) [and the corresponding equation for $\delta \mu$ given by Eq. (21)] are much simpler, while correctly describing all mentioned effects.

\section{CRITICAL RIPPLE AMPLITUDE}

We proceed to determining the critical amplitude of the magnetic field ripple at which the particle motion becomes stochastic. In this section, we will neglect the effect of Garren kicks, considering only the Lagrangian perturbation $\delta \mathcal{L}_{2}$ given by Eq. (32). Moreover, we will disregard the imaginary resonances possible for the perturbation $\delta \mathcal{L}_{1}$ describing the field ripple.

The KAM theory states that the presence of a sufficiently weak perturbation does not change qualitatively the behavior of a Hamiltonian system near most of invariant tori (see, e.g., an overview in Ref. ${ }^{19}$ for a more rigorous discussion of the topic). Most tori are slightly distorted, but their topology remains unchanged, which means that the average effect of the perturbation for a long period of time vanishes. The exceptions are the resonant tori, which in our case are the KAM tori satisfying the resonance condition

$$
\sum_{j=1}^{3} n_{j} \omega_{j}=0
$$

for some integer $n_{j}, j=1,2,3$. On such tori, the effect of the perturbation is secular, i.e., accumulates in time, in linear approximation. Nonlinear consideration shows that the effect of a resonance is determined by the competition between the perturbation magnitude and the "detuning" from the resonance, i.e., the change of the frequencies because of the deviation from the resonant torus. When the perturbation amplitude is relatively small, the modification of the phase-space topology consists only in the appearance of resonance islands in the vicinity of each resonance, the deviations of resonant trajectory on large time intervals being higher than that of nonresonant ones but still restricted. This phenomenon is referred to in the literature as "superadiabaticity". ${ }^{12,13}$ (Another 
term occurring in the literature for the orbital motion of particles in resonance islands is "superbanana motion", which is often used even when the particles under discussion are not banana particles.) At higher amplitudes, which approximately correspond to the overlap of the islands of neighboring resonances (the Chirikov stochasticity criterion ${ }^{19}$ ), the trajectories wander stochastically between different resonances. More exactly, such global stochasticity arises when the ratio of the distance between the resonances to the resonance island width exceeds $\pi / 2$ (although the $\pi / 2$ rule, as well as the Chirikov criterion in general, is proven only for certain Hamiltonian systems, in particular, the standard mapping, ${ }^{20}$ it seems to work in many other cases, see, e.g., Ref. ${ }^{21}$ ).

Thus, the critical perturbation amplitude that is required for the transition of a Hamiltonian system from the superadiabatic behavior to global stochasticity can be found by studying the islands produced by separate resonances. For this purpose, we generalize the approach proposed in Refs. ${ }^{18,22}$. The main idea of the approach is that the phase-space variables can be divided in the vicinity of each resonance $\sum_{j=1}^{3} n_{j} \omega_{j}=0$ into "slow" variables $\left(J_{1}, J_{2}, J_{3}\right.$, and $\left.\sum_{j=1}^{3} n_{j} \varsigma_{j}\right)$ and "fast" ones (two other independent angular coordinates). This, first, opens a possibility to simplify the description of the system by averaging over the fast variables and, second, indicates the existence of an adiabatic invariant associated with such averaging (we refer to it as "a resonance adiabatic invariant"). It turns out that the level contours of the resonance invariant characterize the structure of the resonance island. Hence, the invariant can be used for finding the island width.

To implement this program, we introduce new canonical angular coordinates by the linear transformation

$$
\alpha=\mathrm{Q} \varsigma,
$$

where $\varsigma=\operatorname{col}\left(\varsigma_{1}, \varsigma_{2}, \varsigma_{3}\right), \alpha$ is the vector of the new angles, and

$$
\mathbf{Q}=\left\|\begin{array}{ccc}
n_{1} & n_{2} & n_{3} \\
0 & 1 & 0 \\
1 & 0 & 0
\end{array}\right\| .
$$


One can see from Eqs. (38)-(40) and (50) that the coordinate $\alpha_{1}$ is chosen so that it is constant at the considered resonance. The Lagrangian $\mathcal{L}_{g c}$ then takes the form

$$
\mathcal{L}_{g c}=\mathbf{I} \cdot \dot{\alpha}-\mathcal{E}
$$

where $\mathbf{I}=\left(\mathrm{Q}^{T}\right)^{-1} \mathbf{J}, \mathbf{J}=\operatorname{col}\left(J_{1}, J_{2}, J_{3}\right)$. Without loss of generality, we will consider $I_{2}$ as a function of the other action variables and $\mathcal{E}$. As shown in Appendix B, in a vicinity of the resonance there exists a small coordinate transformation $\left(\alpha_{1}, \alpha_{2}, \alpha_{3}, I_{1}, I_{3}, \mathcal{E}\right) \rightarrow$ $\left(\bar{\alpha}_{1}, \bar{\alpha}_{2}, \bar{\alpha}_{3}, \bar{I}_{1}, \bar{I}_{3}, \mathcal{E}\right)$ such that the Lagrangian of the motion in the rippled field, $\mathcal{L}=$ $\mathcal{L}_{g c}+\delta \mathcal{L}_{2}$, in the new coordinates is free of fast oscillations and has the form

$$
\mathcal{L}=\bar{I}_{1} \cdot \dot{\bar{\alpha}}_{1}+I\left(\bar{\alpha}_{1}, \bar{I}_{1}, \bar{I}_{3}, \mathcal{E}\right)+\bar{I}_{3} \cdot \dot{\bar{\alpha}}_{3}-\mathcal{E}
$$

where

$$
I\left(\bar{\alpha}_{1}, \bar{I}_{1}, \bar{I}_{3}, \mathcal{E}\right)=I_{2}\left(\bar{I}_{1}, \bar{I}_{3}, \mathcal{E}\right)+\tilde{I}\left(\bar{\alpha}_{1}, \bar{I}_{1}, \bar{I}_{3}, \mathcal{E}\right)
$$

$I_{2}=J_{2}(P, \mu, \mathcal{E})-\left(n_{2} / n_{3}\right) P[$ as follows from Eq. (52)];

$$
\tilde{I}=\frac{1}{4 \pi^{2} n_{3}^{2}} \int_{-n_{3} \pi}^{n_{3} \pi} d \varsigma_{10} \int_{L\left(\varsigma_{10}, \alpha_{1}, P, \mu, \mathcal{E}\right)} d \mathbf{x} \cdot \frac{e}{c} \tilde{\mathbf{A}}(\mathbf{x})
$$

$L\left(\varsigma_{10}, \alpha_{1}, P, \mu, \mathcal{E}\right)$ is the curve in the phase space determined by the equations $-n_{3} \pi \leq$ $\varsigma_{2} \leq n_{3} \pi, \varsigma_{1}=\varsigma_{10}+\left(\omega_{1} / \omega_{2}\right) \varsigma_{2}, \varsigma_{3}=\left(\alpha_{1}-n_{1} \varsigma_{1}-n_{2} \varsigma_{2}\right) / n_{3}, P=$ const, $\mu=$ const, $\mathcal{E}=$ const; the parameters $\alpha_{1}$ and $\theta_{10}$ determine the perturbation phase along the curve and the gyrophase at the equatorial plane, respectively. In other words, $L\left(\varsigma_{10}, \alpha_{1}, P, \mu, \mathcal{E}\right)$ is a trajectory of a particle moving with the frequency $\omega_{3}$ modified to satisfy the resonance condition exactly. At the resonance torus, these curves coincide with actual unperturbed trajectories.

Equation (54) presents the Lagrangian of the "slow" motion averaged over fast variables. One can see that $I$ (as well as $\bar{I}_{3}$ and $\mathcal{E}$ ) is a constant of motion of the Euler-Lagrange equations resulting from this Lagrangian. On the other hand, the difference between the coordinates $\left(\bar{\alpha}_{1}, \bar{\alpha}_{2}, \bar{\alpha}_{3}, \bar{I}_{1}, \bar{I}_{3}\right)$ and $\left(\alpha_{1}, \alpha_{2}, \alpha_{3}, I_{1}, I_{3}\right)$ can result only in adiabatic variations of the "non-averaged" variables $\left(\alpha_{1}, \alpha_{2}, \alpha_{3}, I_{1}, I_{3}\right)$. Due to this, $I$ given by Eqs. $(55)$ and 
considered as a function of $\left(\alpha_{1}, \alpha_{2}, \alpha_{3}, I_{1}, I_{3}\right)$ is a new adiabatic invariant - the resonance adiabatic invariant that we have wanted to obtain.

Symmetry properties of Eq. (54) enable us to recover the characteristics of the Eq. (6). Indeed, as mentioned above, $\bar{I}_{3}$ and $\mathcal{E}$ are constants of motion of Eq. (54) (because of the independence of the Lagrangian on $\alpha_{3}$ and $t$, respectively). This means that the resonant motion described by Eq. (54) can result in nonadiabatic changes of $I_{1}$ but adiabatically conserves $I_{3}$. Writing $I_{3}$ in terms of $P=J_{3}$ and $\mu=e J_{1} /(M c)$, we arrive at Eq. (6).

As we are interested in "slow" motion of particles on times long in comparison with the bounce period, below we will not distinguish between the averaged and non-averaged coordinates and will not use bars over variables as a notation of averaging over $\varsigma_{2}$ and $\varsigma_{3}$.

To proceed further, we need to specify the vector potential of the magnetic field ripple, $\tilde{\mathbf{A}}$. We will take into account only the main toroidal Fourier harmonic of the ripple, assuming that $B_{T}(R, Z, \varphi)=B_{T a}(R, Z)\left[1+\delta_{\text {rip }}(R, Z) \cos (N \varphi)\right]$, where $B_{T}$ is the toroidal field, $B_{T a}$ is the toroidal average of $B_{T}$. The specific design of the toroidal field system in STs (the presence of the central post) leads to the spatial dependence of the ripple amplitude of the form $\delta_{\text {rip }}=\delta_{0} \exp \left(R / L_{\text {rip }}\right)$, where $\delta_{0}$ and $L_{\text {rip }}$ are constants (in NSTX $\delta_{0}=7.07 \times 10^{-9}, L_{\text {rip }}=11.36 \mathrm{~cm}$. As a result, the ripple is negligible at the inner circumference, near the central post. We choose the gauge so that $\tilde{A}_{R}=0$, where the subscript denotes the covariant component. As the exponential variation of $\delta_{\text {rip }}$ is much stronger than the variation of $B_{T a}$,

$$
\tilde{A}_{Z}=\int_{0}^{R} d R \delta_{r i p} B_{T a} \cos (N \varphi) \approx L_{r i p} \delta_{r i p} B_{T a} \cos (N \varphi) .
$$

For the same reason, because the dependence of $\bar{B}_{T a}$ is much weaker than the dependence of $\delta_{\text {rip }}$ on $R$, the ripple affects the $R$ component of $\mathbf{B}$ much stronger that the $Z$ component. Therefore, we neglect $\tilde{A}_{\varphi}$, whence $\tilde{\mathbf{A}} \cdot d \mathbf{x} \approx \tilde{A}_{Z} d Z$. We also assume that $\epsilon / q \ll 1$. Then the deviation of the Larmor circles from the vertical plane and the contribution of $v_{\|}$to $d Z$ are negligible, and the main contribution to $\tilde{A}_{Z} d Z$ comes from the gyromotion. It is more convenient for the calculations in this section to set $\hat{\mathbf{e}}_{1}=\nabla R$ and $\hat{\mathbf{e}}_{2}=\nabla Z$. Then the gyrophase is defined so that $R=\bar{R}+\rho_{\perp} \cos \Phi, Z=\bar{Z}-\rho_{\perp} \sin \Phi$, where bars over 
quantities indicate that they are taken at the guiding center, $\rho_{\perp}=v_{\perp} / \omega_{B}$. Substituting these expressions into Eq. (56), setting $d Z \approx-\rho_{\perp} \cos \Phi d \Phi$, and using Eq. (57), we obtain

$$
\begin{aligned}
\tilde{I} & =-\frac{1}{4 \pi^{2} n_{3}^{2}} \int_{-n_{3} \pi}^{n_{3} \pi} d \varsigma_{10} \int_{L\left(\varsigma_{10}, \alpha_{1}, P, \mu, \mathcal{E}\right)} d \Phi L_{r i p} \bar{\delta}_{r i p} \bar{B}_{T} \rho_{\perp} \\
& \times \cos \Phi \exp \left(\frac{\rho_{\perp}}{L_{\text {rip }}} \cos \Phi\right) \cos (N \varphi) .
\end{aligned}
$$

Expressing $\Phi$ and $\varphi$ in Eq. (58) in terms of canonical coordinates via Eq. (34), expanding the integrand in a Fourier series and using the definition of $L\left(\varsigma_{10}, \alpha_{1}, P, \mu, \mathcal{E}\right)$, we find

$$
\begin{aligned}
\tilde{I} & =-\frac{1}{4 \pi^{2} n_{3}^{2}} \sum_{l, s=-\infty}^{\infty}\left\{\Upsilon_{l s} \int_{-n_{3} \pi}^{n_{3} \pi} d \varsigma_{10} \exp \left[i\left(\frac{N n_{1}}{n_{3}}+l\right) \varsigma_{10}\right]\right. \\
& \left.\times \int_{-n_{3} \pi}^{n_{3} \pi} d \varsigma_{2} \frac{\omega_{1}}{\omega_{2}} \exp \left[i\left(\frac{N n_{1}}{n_{3}}+l\right) \varsigma_{2}+i\left(\frac{N n_{2}}{n_{3}}-s\right) \varsigma_{2}\right]+\text { c.c. }\right\} .
\end{aligned}
$$

where "c.c." means complex conjugate,

$$
\Upsilon_{l s}(P, \mu, \mathcal{E})=\frac{e L_{r i p}}{4 c \omega_{b}} \frac{1}{2 \pi} \oint d \varsigma_{2} \bar{\delta}_{r i p} \bar{B}_{T} v_{\perp} a_{l}\left(\frac{\rho_{\perp}}{L_{\text {rip }}}\right) \exp (-i N \zeta)
$$

$\left.a_{l}(x)=\left[\mathbf{I}_{|l-1|}\right)(x)+\mathrm{I}_{|l+1|}(x)\right] / 2, \mathrm{I}_{j}(x)$ are modified Bessel functions of the first kind, $\zeta=$ $\zeta\left(\varsigma_{2}, P, \mu, \mathcal{E}\right)=v+i l \varpi / N+i s \varsigma_{2} / N$. One can see that the integral in Eq. (59) vanishes for given $l$ and $s$ unless $n_{1} / n_{3}=-l / N, n_{2} / n_{3}=s / N$. Thus, the effect of the resonance described by Eq. (50) can be non-zero only when this equation coincides with the global resonance condition given by Eq. (4) up to a factor. Therefore, we will consider without loss of generality that $n_{1}=l, n_{2}=-s, n_{3}=-N$.

For large $N$, the factor $\exp (-i N \zeta)$ in the integrand of Eq. (60) is typically a rapidly oscillating function of $\varsigma_{2}$. It is known that the main contribution to such integrals comes from the vicinities of the points of stationary phase, i.e., the points where

$$
-i N \frac{\partial \zeta}{\partial \varsigma_{2}} \equiv-N \frac{\partial v}{\partial \varsigma_{2}}+l \frac{\partial \varpi}{\partial \varsigma_{2}}+s=0
$$

Using Eqs. (4) and (34), one can show that Eq. (61) at the exact global resonance is equivalent to the condition of local resonance [Eq. (5)]. Thus, the jumps of the mentioned integral in Eq. (60) reflect the well-known jump-like behavior of particles near the points of the local resonance. 
We observe that the integral in Eq. (60) contains only the effect of local resonances with the same value of $l$ as in the global resonance condition, which corresponds to our discussion of the interplay of global and local resonances in Sec. II. This enables us to make an important conclusion that the width of resonance islands with some given $l$ is not affected by particle jumps caused by local resonances with other values of $l$. Typically, there are two points of the local resonance for a given $l$ on a particle orbit. For instance, a banana particle in NSTX launched at the equatorial plane with $\chi>0.6$ passes through two points of the $l=2$ resonance, two points of the $l=1$ resonance, two points of the $l=0$ resonance, and two points of the $l=-1$ resonance for each bounce. However, if we are interested in the CRI diffusion with, e.g., $l=1$, only one pair of the local resonance points appears in the corresponding integral in Eq. (60). There exist orbits with four $l=0$ resonance points $(\dot{\varphi}=0)$ in high- $\beta$ discharges of $\mathrm{STs}_{\mathrm{s}}{ }^{8}$ but such orbits are not numerous. ${ }^{23}$ One can expect a similar situation for other $l$, too. Therefore, we assume that there are only two points of local resonance on each orbit. Then the mathematical description of the resonant motion for different $l$ turns out to be rather similar, and we can generalize to any $l$ the analysis of the transition to chaos made for the case of the GWB diffusion in Ref. ${ }^{21}$.

Keeping only the resonance term in Eq. (59) and applying the stationary-phase method to Eq. (60), we can write Eq. (59) as follows:

$$
\tilde{I}=-\left.\frac{e L_{r i p}}{(2 \pi N)^{1 / 2} c} \sum_{j=1}^{2} \frac{\bar{\delta}_{r i p} \bar{B}_{T} v_{\perp}}{|\Xi|^{1 / 2}} a_{l}\left(\frac{\rho_{\perp}}{L_{r i p}}\right) \cos \left[\alpha_{1}+N \zeta-\operatorname{sgn}(\zeta) \frac{\pi}{4}\right]\right|_{\varsigma_{2}=\varsigma_{2 j}}
$$

where $\varsigma_{2}=\varsigma_{2 j}, j=1,2$, are the coordinates of the points of the local resonance,

$$
\Xi \equiv \omega_{b}^{2} \frac{\partial^{2} \zeta}{\partial \varsigma_{2}^{2}}=\frac{v^{2}}{2 q R^{2}} \frac{\partial \ln B}{\partial \theta}\left(1+\frac{l^{2} R^{2}}{N^{2} \rho^{2}}\right)+\frac{l \omega_{B}}{N} \frac{d \ln R}{d t} .
$$

For $l=0$, Eq. (62) is in agreement with an equation of motion in the vicinity of the resonance obtained from other considerations in $\operatorname{Ref.}^{21}$ [Eq. (21) of the mentioned work].

Below we restrict ourselves to the case of the up-down symmetry. Then, expanding $I_{2}$ in a series near the resonance and taking into account that $\partial I_{2} / \partial I_{1}=0$ at the resonance, we obtain from Eqs. (55) and (62) the following expression for the resonance invariant: 


$$
I=\left.\frac{N}{2 \pi} \varphi_{p}^{\prime}\right|_{I_{1}=I_{1 r}}\left(I_{1}-I_{1 r}\right)^{2}+\frac{1}{\pi} \delta I_{1}\left(I_{1}, I_{3}, \mathcal{E}\right) \cos \left[\frac{N}{2} \varphi_{b}\left(I_{1}, I_{3}, \mathcal{E}\right)\right] \cos \alpha_{1},
$$

where "r" refers to the value at the resonance, prime denotes $\partial / \partial I_{1}=-N \partial / \partial J+$ $(l e / M c) \partial / \partial \mu, \varphi_{b}=2\left|\zeta\left(\varsigma_{2 j}\right)\right|-\pi /(2 N), \varphi_{p}=(\pi / N)\left(\partial I_{2} / \partial I_{1}-s\right)=\pi\left(\omega_{\varphi}-l\left\langle\omega_{B}\right\rangle / N\right) / \omega_{b}$,

$$
\delta I_{1}=-\left.\left(\frac{2 \pi}{N}\right)^{1 / 2} \frac{e L_{r i p}}{c}\left[\frac{\bar{\delta}_{r i p} \bar{B}_{T} v_{\perp}}{|\Xi|^{1 / 2}} a_{l}\left(\frac{\rho_{\perp}}{L_{r i p}}\right)\right]\right|_{\varsigma_{2}=\varsigma_{2 j}} .
$$

Note that one can attribute a graphic physical meaning to the quantities $\varphi_{b}, \varphi_{p}$, and $\delta I_{1}$. Let $t_{1}, t_{2}, t_{3}, \ldots$ be consecutive instants of the local resonance, so that $\varsigma_{2}\left(t_{1}\right)=\varsigma_{21}$, $\varsigma_{2}\left(t_{2}\right)=\varsigma_{22}=-\varsigma_{21}, \varsigma_{2}\left(t_{3}\right)=\varsigma_{21}$, etc. Then it follows from the definitions of $\varphi_{b}$ and $\varphi_{p}$ that the phase of the resonant ripple-particle interaction, $\sigma \equiv N \varphi-l \Phi-\operatorname{sgn}(\zeta) \pi / 4=$ $N \varsigma_{3}-l \varsigma_{1}+N \zeta-\operatorname{sgn}(\zeta) \pi / 4$, evolves between the resonance events as follows:

$$
\begin{gathered}
\sigma\left(t_{2 j+1}\right)=\sigma\left(t_{2 j}\right)+N \varphi_{p}+N \varphi_{b}, \\
\sigma\left(t_{2 j+2}\right)=\sigma\left(t_{2 j+1}\right)+N \varphi_{p}-N \varphi_{b},
\end{gathered}
$$

where we have assumed for definiteness that $\zeta\left(\varsigma_{21}\right)>0, \zeta\left(\varsigma_{22}\right)<0$. In this sense, $\varphi_{b}$ and $\varphi_{p}$ play the same role as the corresponding quantities introduced in Refs. ${ }^{1,21}$ do for the GWB diffusion (in particular, for $l=0$ these quantities as they are defined here exactly coincide with those in Refs. $\left.{ }^{1,21}\right)$. It turns out that $\delta I_{1}$ is the characteristic jump of $I_{1}$ for one local resonance event, which justifies the notation chosen for this quantity. Indeed, in the averaged description [Eq. (54)] the jumps of $I_{1}$ can be found as the variation of $I_{1}$ for half a bounce period. An equation for $\dot{I}_{1}$ can be obtained from Eq. (54) by varying $\alpha_{1}$. Substituting Eq. (65) for $D$, we obtain

$$
\int_{t}^{t+\tau_{b} / 2} d t \dot{I}_{1}=-\delta I_{1} \cos \left[\frac{N}{2} \varphi_{b}\left(I_{1}, I_{3}, \mathcal{E}\right)\right] \sin \alpha_{1} .
$$

The structure of the resonance islands for the particle resonance with the ripple causing the GWB diffusion is known ${ }^{21}$ to depend crucially on the ratio of $\varphi_{b}^{\prime} / \varphi_{p}^{\prime}$. At small $\varphi_{b}^{\prime} / \varphi_{p}^{\prime}$, the resonance islands constructed by the Poincaré mapping technique resemble the classical Chirikov islands studied in the theory of nonlinear resonance ${ }^{19}$ the island width 
depending on the ripple amplitude as $\delta_{\text {rip }}^{1 / 2}$. When $\varphi_{b}^{\prime} / \varphi_{p}^{\prime} \gg 1$, the Poincaré mappings show the appearance of additional resonance islands aside from the main island. The arising structure was called in Ref. ${ }^{21}$ "the resonance web". The onset of the global stochasticity coincides with the overlap of the webs of neighboring resonances (allowing for the $\pi / 2$ rule), the extent of which in this case is $\propto \delta_{\text {rip }}$. The difference of the mechanisms leading to stochasticity in the two mentioned cases gives grounds to speak about two different routes to chaos. ${ }^{21}$

This difference in the island structure for different magnitudes of $\varphi_{b}^{\prime} / \varphi_{p}^{\prime}$ is reflected by the level contours of the invariant given by Eq. (64). When $\varphi_{b}^{\prime}$ is relatively small, the $\cos \varphi_{b}$ factor in Eq. (64) can be considered constant. Then the levels of line of the invariant describe a conventional Chirikov island ${ }^{19}$ with the half width $\Delta I_{1 c}=2\left[\delta I_{1} /\left(N \varphi_{p}^{\prime}\right)\right]^{1 / 2}$, see Fig. 2. When, on the contrary, $\varphi_{b}^{\prime}$ is large so that $Q \equiv(N / 2) \phi_{b}^{\prime} \Delta I_{1 c}=\phi_{b}^{\prime}\left(N \delta I_{1} / \phi_{p}^{\prime}\right)^{1 / 2} \gg$ 1, the second term in the right-hand side of Eq. (64) is a rapidly oscillating function of $I_{1}$. Then the level contours of $I$ show chains of additional driven-resonance islands ("resonance web") on both sides of the resonance (see Fig. 3). An analysis of the local extrema of $I$, which essentially repeats the analysis of the fixed points of the mapping in Ref. ${ }^{21}$, shows that the first driven islands appear at $Q \simeq 2$. Comparing Fig. 3 (b) with Fig. 7 of Ref. ${ }^{21}$, one can see that the resonance invariant reproduces the structure of the resonance islands obtained with Poincaré mappings well enough. Thus, it can be used for evaluating the island width and finding the amplitude threshold of the transition to stochasticity.

In the limit case of $\varphi_{b}^{\prime} \gg \varphi_{p}^{\prime}$, the chain of the driven islands ends when the derivative of the first term in Eq.(64) exceeds that of the second one. Hence, we obtain the following expression for the half-width of the resonance web (cf. Ref. $\left.{ }^{21}\right)$ :

$$
\Delta I_{1}=\frac{D \varphi_{b}^{\prime}}{2 \varphi_{p}^{\prime}} .
$$

The distance in $I_{1}$ between the neighboring resonances, $N \omega_{\varphi}+s \omega_{b}-l\left\langle\omega_{B}\right\rangle=0$ and $N \omega_{\varphi}+(s+1) \omega_{b}-l\left\langle\omega_{B}\right\rangle=0$, equals to $\pi /\left(N \varphi_{p}^{\prime}\right)$. Applying the $\pi / 2$ rule, we obtain the critical value of $\delta I_{1}$ (the stochasticity threshold): 


$$
\delta I_{1 c r i t}=\frac{2}{N \varphi_{b}^{\prime}}
$$

An expression for $\delta I_{1 c r i t}$ in the limit case, $\varphi_{p}^{\prime} \gg \varphi_{b}^{\prime}$ were obtained for the GWB diffusion in Ref. ${ }^{21}$, allowing for the up-down asymmetry. However, it seems convenient for practical purposes to use the approximate stochasticity threshold

$$
\delta I_{1 c r i t}=\frac{2}{N\left(\left|\varphi_{p}^{\prime}\right|+\left|\varphi_{b}^{\prime}\right|\right)}
$$

which was shown to work in a wide range of magnitudes of $\varphi_{b}^{\prime} / \varphi_{p}^{\prime}$ by direct calculations of Poincaré mappings. ${ }^{24}$ The full mathematical similarity of the $l=0$ and $l \neq 0$ cases revealed in the above analysis enables us to use this equation for the stochasticity threshold for the CRI diffusion, too. From Eqs. (65) and (71) we obtain the following stochasticity criterion:

$$
\bar{\delta}_{r i p}>\delta_{c r i t} \equiv \frac{(2|\Xi|)^{1 / 2} c}{(\pi N)^{1 / 2} e \bar{B} v_{\perp} L_{r i p} a_{l}\left(\rho_{\perp} / L_{\text {rip }}\right)\left(\left|\varphi_{p}^{\prime}\right|+\left|\varphi_{b}^{\prime}\right|\right)}
$$

where all quantities are taken at the local resonance point. In particular, this criterion agrees with previously obtained criteria for the GWB diffusion $(l=0)$. Indeed, assuming that $\rho \ll L_{\text {rip }}$, we have $L_{\text {rip }} a_{0}\left(\rho_{\perp} / L_{\text {rip }}\right)=\rho / 2$. For $l=0$, primes become $-(N c / e) \partial / \partial \psi$. Taking $\theta \approx \pi / 2$ at the turning point, we write Eq. (63) as $\Xi=v^{2} /\left(2 q R^{2}\right)$, and Eq. (72) reduces to the criterion of Ref. ${ }^{1}$. When $\rho>L$, the factor $a_{0}\left(\rho_{\perp} / L\right)$ takes account of the difference between the ripple magnitude at the guiding center and that averaged over the Larmor circle (cf. Ref. ${ }^{26}$ ). As energetic ions in modern ST are characterized by large Larmor radius exceeding $L_{\text {rip }}$, this correction may be considerable. For instance, this results in a decrease of $\delta_{\text {crit }}$ by a factor of 1.5 for $\rho / L_{\text {rip }}=2$, as is the case for $80-\mathrm{keV}$ deuterons in NSTX. Note that Eq. (72) may not agree with the stochasticity threshold for the CRI diffusion obtained in Refs. ${ }^{2,25}$, especially for large magnitudes of $N \rho / R$, because the criterion of Refs. ${ }^{2,25}$ was obtained without taking account of jumps in $P$.

High $\beta$ attributed to modern and future STs affects both $\varphi_{b}^{\prime}$ and $\varphi_{p}^{\prime}$. The change of $\varphi_{p}^{\prime}$ is a consequence of the fact that high $\beta$ changes the precession frequency of trapped particles (even the direction of the precession can be changed) ${ }^{27}$ and strongly enhances 
the precession of circulating particles. ${ }^{7}$ To demonstrate the effect of high $\beta$ on $\varphi_{b}^{\prime}$, we note that in the case of the GWB diffusion, $\varphi_{b}^{\prime} \propto\left[\hat{s} \theta-r B_{r}^{\prime} / B_{\theta}^{\prime}\right]$, where $B_{r}^{\prime} \equiv \partial B / \partial r$, $B_{\theta}^{\prime} \equiv \partial B / \partial \theta, r \propto \sqrt{\psi}$, and $\hat{s}$ is the magnetic shear. In CTs with low $\beta$ the first term dominates for $\theta \sim 1$. In contrast to this, the second term dominates at high $\beta$. Indeed, when $\theta \approx \pi / 2$, the magnetic field can be approximated in the region not very close to the edge as $B \propto B_{0}\left(1+\alpha \epsilon^{2}\right)$, where $\alpha$ is a parameter small for low $\beta$ and well exceeding unity at high $\beta,{ }^{27}$ which leads to a large radial derivative of $B$ for $\theta \sim 1$ and high $\beta$.

\section{EFFECT OF STOCHASTIC DIFFUSION ON CONFINEMENT OF ENERGETIC PARTICLES}

\section{A. Ripple-induced transport}

To compare the relative role of various mechanisms of stochastic diffusion in the particular case of 80-keV ions in NSTX, we have calculated the stochastic region and diffusion trajectories for various $l$, using a simplified model of the magnetic field. The calculations show that the region of the GWB stochastic loss is relatively small, and the orbits of most particles with banana tips in the region probably cross the wall even without ripple. The loss regions of the $l=1$ and $l=2$ diffusion are more extensive.

It is of importance to know which energetic particles can be lost during the stochastic diffusion. It is clear that a particle can be lost if its local resonance point can reach the wall along a trajectory determined by Eq. (18), while staying in the stochasticity region. In the case of a low- $\beta$ plasma, all trajectories of resonance points of the GWB diffusion, are vertical lines and, thus, strike the wall. In contrast to this, the trajectories of resonance points of the CRI diffusion are approximately ellipses; therefore, the loss region shrinks with the increase of the resonant pitch angle, see Eq. (19). In high- $\beta$ plasmas, some trajectories may lie within the plasma even for the GWB diffusion. On the other hand, it is clear that not all particles can take part in the stochastic diffusion because the particle must satisfy the condition of local resonance. For the GWB diffusion, this condition singles out all trapped particles (more exactly, all particles with $v_{\|}=0$ at some 
point of the orbit). The situation in the case of the CRI diffusion is more complicated. It turns out that the fraction of the phase space occupied by resonant particles increases with the decrease of the resonance pitch angle, $\chi_{r}=l R /(N \rho)$, and is considerable in NSTX. Indeed, the overall number of the particles that pass through the given value of $\chi=\chi_{r}$ is approximately proportional to the variation of $\chi$ on a trajectory of a single particle, which can be estimated for a circulating particle as $\Delta \chi=2 \epsilon\left(1-\chi_{r}^{2}\right) / \chi_{r}$ (we have used the equation $\chi=\left(1-\lambda R_{0} / R\right)^{1 / 2}$. Taking, for instance, $\epsilon=0.5$, we find that $\Delta \chi \sim 1$ for the $l=1$ - and $l=2$-resonant particles in $\operatorname{NSTX}\left(\chi_{r}=0.3\right.$ and 0.6 , respectively).

The CRI diffusion is not necessarily harmful. It improves the confinement of the injected ions with $v_{\|}>0$ (which have the maximum radial excursion at $\theta=0$ ) and $\lambda \ll 1$, leading to their inward motion (because for these particles $\Delta \lambda>0$ ), see Eq. (15).

The CRI diffusion may result in orbit transformations, including transformations of circulating particles into trapped ones (CT transformations) and vice versa (TC transformations). In order to see it, we write $v_{\|}= \pm v\left(1-\lambda B / B_{0}\right)^{1 / 2}$, from which it follows that CT and TC transformations may occur when $B \Delta \lambda+\lambda \Delta B>0$ and $B \Delta \lambda+\lambda \Delta B<0$, respectively. As follows from Eq. (16), $\operatorname{sgn}(\Delta r)=\operatorname{sgn}(l \Delta \lambda)$, which means that the effect of a change of $\lambda$ either enhances the effect of $\Delta B$ (for $l>0$ ) or competes with it (for $l<0)$. In the latter case, $\Delta \lambda$ can result in TC transformations during the outward motion of particles provided that

$$
\frac{B \Delta \lambda}{\lambda \Delta B}=\frac{2 l k\langle r\rangle R}{N q \rho^{2} \lambda}>1
$$

where we have used the relationship $\Delta B / B \sim \delta r / R_{0}$. The transformations due to the $l<0$ diffusion result in a sharp increase (for CT transformations) or decrease (for TC transformations) of the maximum $r$ of the particle orbit.

A loss of particles because of the stochastic diffusion may occur through an "estafette" of resonances with various $l$. In particular, the CRI diffusion of circulating particles that results in the CT orbit transformation may be followed by the GWB diffusion.

The confinement of particles may severely deteriorate if the particles satisfy the stochasticity criterion for several $l$ simultaneously. As such particles are able to move 
along several families of characteristics [Eqs. (6)] corresponding to different $l$, their motion is not constrained by each of them. In this case, even well circulated particles can diffuse from the plasma.

Let us compare the characteristic time of the particle loss due to stochasticity, $\tau_{D}$, with the slowing-down time, $\tau_{s}$. Taking the characteristic jump of $I_{1}$ during a single local resonance event, $\delta I_{1}$, from Eq. (65) and allowing for $\delta I_{2}=\delta I_{3}=0$, we find the characteristic jump of $P, \delta P=N \delta I_{1}$. We take $\Xi=-\epsilon v^{2} \sin \theta /\left(2 q R^{2}\right)$, neglecting the rest of the terms in Eq. (63). The radial particle jump can then be estimated as

$$
\Delta r=\frac{c q}{e B r k} \delta P=\frac{2 \bar{\delta}_{r i p}}{k}\left[\frac{q^{3} N \pi\left(1-\chi^{2}\right)}{\epsilon^{3} \sin \theta}\right]^{1 / 2} L_{\text {rip }} a_{l}\left(\frac{\rho_{\perp}}{L_{\text {rip }}}\right)
$$

whence we evaluate the time required for a particle to pass the distance $d$ as

$$
\tau_{D}=\frac{\pi d^{2}}{\omega_{b}(\delta r)^{2}}=\frac{d^{2} k^{2} \epsilon^{3} \sin \theta}{4 \omega_{b} \bar{\delta}_{r i p}^{2} q^{3} N L_{r i p}^{2} a_{l}^{2}\left(\rho_{\perp} / L_{r i p}\right)\left(1-\chi^{2}\right)}
$$

where all quantities are taken at the local resonance point. Equation (75) is valid for $\theta>\theta_{\text {min }}$ with $\sin \theta_{\text {min }}=(\pi / \epsilon)\left(\omega_{b} / \omega_{B}\right)$ for the circulating particles. When $\theta<\theta_{\text {min }}$, $\theta$ must be replaced by $\theta_{\min }$ in Eq. (75). Taking $d=30 \mathrm{~cm}$ and parameters of $80-\mathrm{keV}$ deuterons in NSTX, we obtain $\tau_{D}=2.4 \times 10^{-2} \mathrm{~s}$, which is higher than $\tau_{s}$ in current NSTX experiments $\left(\sim 10^{-2} \mathrm{~s}\right)$. We conclude that the ripple-induced stochastic diffusion can hardly play noticeable role in NSTX until higher plasma temperatures are reached.

\section{B. Transport in an axisymmetric field}

The diffusion length $\left(L_{D}\right)$ of an energetic ion can be estimated as $L_{d}=\sqrt{D \tau_{\epsilon}}$, where $\tau_{\epsilon}$ is the slowing down time, $D \sim(\delta r)^{2} / \tau_{b}$ is the diffusion coefficient. Taking into account that $\delta \lambda=0$ for $\lambda=0$ [see Eq. (26)], we use the characteristic magnitude $\lambda \approx \lambda_{\text {max }} / 2$, where $\lambda_{\max }=1 /(1+\epsilon)$ is the pitch angle of the marginally circulating particles. Then we obtain:

$$
L_{d}=\rho\left(\epsilon^{-1}-1\right) \sqrt{\frac{q}{8 \pi \chi_{m}} \frac{v \tau_{\epsilon}}{R}} \frac{\delta \lambda}{k} .
$$


For instance, for $80-\mathrm{keV} \mathrm{D}$ ions in NSTX with $\rho=20 \mathrm{~cm}, r=a / 2=33 \mathrm{~cm}, \epsilon=0.4$, $q=1.5, \tau_{\epsilon}=0.01 \mathrm{~s}, \lambda=0.36, \delta \lambda=1.8 \times 10^{-3}$, we obtain $L_{d}=1.5 \mathrm{~cm}$. Thus, the effect of the radial diffusion in the considered case is negligible. But maybe the pitch-angle scattering itself can affect the particle confinement by resulting in orbit transformations of a large fraction of the particles? This effect could be important in machines where the width of the particle orbits $\left(\Delta r_{b}\right)$ is comparable with the plasma radius, so that the particles could be pushed into the loss cone by the orbit transformations.

Let us analyze this possibility.

One can see that $\langle r\rangle$ changes negligibly during the transformation (this can be easily shown by using the equation $P=$ const and taking into account that the precession velocities of the marginally trapped and marginally circulating particles are approximately equal). But $\delta r_{\max } \sim \Delta r_{b} / 2$, which can result in escape of the particles to the loss cone. However, one usually chooses such a direction of the injection that the transformation of circulating particles to trapped ones is accompanied by a jump of $r_{\min }$ inside the plasma, whereas $\delta r_{\max }=0$, in which case the transformation improves the confinement. To see the role of the orbit transformation for particles with isotropic velocity distribution (e.g., alpha particles), we estimate the local fraction of the circulating particles shifted outwards/inwards $\left(\nu_{l}\right)$ as follows. We use the fact that when the distribution is isotropic, $\nu_{l}=\left|\Delta \chi_{m}\right| / 2$, where $\left|\Delta \chi_{m}\right|=|\Delta \lambda|(1-\epsilon) /\left(2\left|\chi_{m}\right|\right.$ ) (because $\left.\left|\chi_{m}\right|=\sqrt{1-\lambda(1-\epsilon)}\right), \Delta \lambda$ is the diffusion in $\lambda$ for the slowing-down time given by

$$
\Delta \lambda \sim \delta \lambda \sqrt{\frac{\tau_{\epsilon}}{\tau_{b}}} .
$$

We obtain:

$$
\nu_{l}=\frac{(1-\epsilon)}{4 \chi_{m}} \sqrt{\frac{\tau_{\epsilon}}{\tau_{b}}} \delta \lambda .
$$

Taking again $\epsilon=0.4, \lambda=0.36$, and $\tau_{\epsilon} / \tau_{b}=10^{4} /(2 \pi)$, we have $\delta \lambda=1.8 \times 10^{-3}, \chi_{m}=0.88$, which leads to $\nu_{l}=1.2 \%$.

Thus, the Garren effect cannot influence energetic ions in the plasma core of NSTX, but one cannot rule out that it will be noticeable at the plasma periphery. 
Note that effects of the nonconservation of $\mu$ in spherical tori were studied in Refs. ${ }^{28,29}$. In the first of them it was found that nonadiabaticity affects only beam ions already following loss orbits and should not have any substantial negative effect on the confinement of the energetic ions in NSTX, which agrees with our conclusion. In contrast to this, in

Ref. ${ }^{29}$ it was claimed that the nonconservation of $\mu$ was expected to affect the confinement of fast ions significantly, in particular, resulting in enhanced radial diffusion, although no estimates of the radial diffusion were done.

\section{SUMMARY AND CONCLUSIONS}

We have analyzed various mechanisms of the stochastic diffusion of energetic ions in STs, the diffusion in both the axisymmetric magnetic field and the rippled field having been studied. The analysis is carried out in a unified approach, in which different mechanisms correspond to different magnitudes of $l(l=0, \pm 1, \pm 2, \ldots)$, where $l$ is the cyclotron harmonic number in the local and global resonances responsible for the interaction between the energetic ions and the magnetic configuration. In this approach, the Garren effect in an axisymmetric field corresponds to $l=1$, but the local resonance, in contrast to the global one, is imaginary, i.e., it occurs in the complex plane. The local and global resonances with $l=0$ are responsible for the GWB diffusion, whereas $l= \pm 1, \pm 2, \pm 3, \ldots$, for the CRI diffusion caused by the ripple.

A Lagrangian formalism for the description of the nonadiabatic effects in the particle motion is developed. Based on this formalism, a general expression for the critical ripple amplitude is obtained, which in a special case of the GWB diffusion of particles with small Larmor radius, $\rho \ll L_{\text {rip }}$, yields the result of Refs. ${ }^{1,24}$. In addition, a formula for a jump of the magnetic moment (Garren effect) is derived, which agrees with that obtained by a different procedure in Refs. ${ }^{9,10}$. Our formula takes into account effects of the plasma pressure and has a simpler form. Therefore, we used it to analyze the Garren effect in NSTX.

We have shown that CRI diffusion affects trapped and marginally circulating particle 
and plays larger role in STs than that in CTs, which is associated with a larger ratio of $\rho^{2} / r^{2}$ in STs. Furthermore, probably, in low- $B$ STs it is more important than the GWB diffusion. The GWB diffusion is weakened by the large toroidal precession. In addition, most particles with the banana tips in the region of the strong ripple are promptly lost. Although $\delta_{\text {crit }}$ decreases due to the finite Larmor radius, this effect is not large. In particular, in NSTX $\delta_{\text {crit }}$ decreases by a factor of 1.5. The CRI diffusion may result in orbit transformations, the circulating particles can be transformed into the trapped ones and vice versa, depending on the sign of $l$. Furthermore, an estafette of the resonances may occur, i.e., various mechanisms of stochastic diffusion may be responsible for the transport of a particle in the plasma core and near the periphery when stochastic regions corresponding to different $l$ partly overlap. The character of diffusion in a region where the stochasticity condition is satisfied for various $l$ simultaneously differs from that in a region of stochasticity dominated by only one resonance.

Estimates of the ratio of the diffusion time of the $80-\mathrm{keV}$ ions to their slowing-down time indicate that the stochastic diffusion associated with the Garren effect and the ripple is not fast enough to deteriorate the confinement of energetic ions in current experiments on NSTX, i.e., the ions slow down before they diffuse over a considerable part of the plasma radius. They show that the diffusion can have a noticeable effect only in the periphery region. The situation may change if experiments deal with plasmas characterized by lower density and higher electron temperature, in which case the slowing-down time of the energetic ions will increase so that it may exceed the diffusion time. One can expect that the loss of energetic ions will then increase. On the other hand, if the stochastic diffusion in current experiments plays a noticeable role, the confinement of the energetic ions can be improved by increasing the plasma density and decreasing the electron temperature (to decrease the ratio $T_{e}^{3 / 2} / n_{e}$, where $T_{e}$ and $n_{e}$ are the electron temperature and density, respectively).

In conclusion, we note that the magnetic field in a design of the spherical torus reactor DTST is rather strong $(B=4.5 \mathrm{~T})$, and the plasma size is rather large $(a=74 \mathrm{~cm}$, $R=134 \mathrm{~cm}) \cdot{ }^{30}$ Therefore, one can expect that the GWB diffusion will dominate the 
stochastic motion of $3.5-\mathrm{MeV}$ alpha particles in this machine.

\section{ACKNOWLEDGMENTS}

The authors would like to thank Dr. S. Jardin for giving parameters of the updated DTST design and Dr. J. Manickam for a numerically calculated NSTX equilibrium. Two

of the authors (Y.K. and Y.Y.) would like to acknowledge the hospitality of the Princeton Plasma Physics Laboratory.

The research described in this publication was made possible in part by the Award No. UP2-2114 of the U.S. Civilian Research \& Development Foundation for the Independent States of the Former Soviet Union and the Government of Ukraine, the U.S. Department of Energy under contract number DE-AC02-76-CHO3073, and the Research Contract No. 10539 of the International Atomic Energy Agency.

\section{APPENDIX A: FIELD LINE CURVATURE IN A CIRCULAR TOKAMAK}

We find the field line curvature to the first order in $\epsilon=r / R_{0} \ll 1$ in a tokamak with $\beta \ll 1$ and a circular cross section. It follows from the equilibrium equation,

$$
(\nabla \times \mathbf{B}) \times \mathbf{B}=4 \pi \nabla p
$$

that the field line curvature, $\kappa=(\hat{\mathbf{b}} \cdot \nabla) \hat{\mathbf{b}}$, can be found as follows:

$$
\kappa=\frac{1}{2 B^{2}} \nabla_{\perp}\left(B^{2}+8 \pi p\right)=\frac{1}{2 B^{2}}\left[\nabla\left(B^{2}+8 \pi p\right)-\hat{\mathbf{b}} \nabla_{\|} B^{2}\right]
$$

whence the scalar curvature is

$$
\kappa=\frac{1}{2 B^{2}}\left[\left|\nabla\left(B^{2}+8 \pi p\right)\right|^{2}-\left|\nabla_{\|} B^{2}\right|^{2}\right]^{1 / 2}
$$

As $\nabla_{\|} B \sim \epsilon \nabla_{\perp} B$, the second term of Eq. (A3) is $\epsilon^{2}$ times smaller than the first one and will be neglected.

An axisymmetric magnetic field can be presented in the form

$$
\mathbf{B}=F(r) \mathbf{e}^{\varphi}+\frac{1}{\sqrt{g}} \mathbf{e}_{\theta} \frac{d \Psi}{d r},
$$


where the coordinate system $(r, \theta, \varphi)$ is taken so that the radial coordinate is constant on each flux surface; $\Psi=\Psi(r)$ is the poloidal magnetic flux; $F(r)=B_{0} R_{0}\left[1+\mathcal{O}\left(\epsilon^{2}+\beta\right)\right]$ is a function of $r$ describing the plasma diamagnetism; $\mathbf{e}_{j}$ and $\mathbf{e}^{j}, j=r, \theta, \varphi$, are the corresponding co- and contravariant base vector; $g$ is the determinant of the metric tensor. One can show that in a circular tokamak to main order in $\epsilon$

$$
g_{\theta \theta}=r^{2}, \quad \sqrt{g}=r R_{0}, \quad g_{r \theta} \sim \epsilon r
$$

where $g_{r \theta}$ and $g_{\theta \theta}$ are the corresponding components of the covariant metric tensor (see, e.g., Ref. $\left.{ }^{31}\right)$. Then the poloidal component of the magnetic field, $B_{p}$, in a circular tokamak [which is described by the second term of Eq. (A4)] is constant over a flux surface to main order,

$$
B_{p}=\left(\frac{g_{\theta \theta}}{g}\right)^{1 / 2} \frac{d \Psi}{d r}=\frac{\epsilon}{q} B_{0}[1+\mathcal{O}(\epsilon)]
$$

where $q$ is the safety factor. From Eqs. (A3), (A4), and (A6), the curvature is

$$
\kappa=\frac{1}{2 B^{2}}\left|\nabla\left(\frac{F^{2}}{R^{2}}+\frac{\epsilon^{2} B^{2}}{q^{2}}+8 \pi p\right)\right|\left[1+\mathcal{O}\left(\epsilon^{2}\right)\right] .
$$

Using Eqs. (A4)-(A6), Eq. (A1) can be written as follows:

$$
8 \pi \frac{d p}{d r}+\frac{1}{R^{2}} \frac{d\left(F^{2}\right)}{d r}+\frac{d}{d r} \frac{\epsilon^{2} B_{0}^{2}}{q^{2}}=-\frac{2 \epsilon^{2}}{r q^{2}} B_{0}^{2}+\mathcal{O}\left(\epsilon^{3} \frac{B_{0}^{2}}{r}\right)
$$

whence

$$
\nabla\left(\frac{F^{2}}{R^{2}}+B_{0}^{2} \frac{\epsilon^{2}}{q^{2}}+8 \pi p\right)=-2 \frac{F^{2}}{R^{3}} \nabla R-2 \frac{\epsilon^{2} B_{0}^{2}}{r q^{2}} \nabla r+\mathcal{O}\left(\epsilon^{3} \frac{B_{0}^{2}}{r}\right)
$$

Comparing Eqs. (A7) and (A9) and neglecting second-order terms, we find

$$
R_{c}=\kappa^{-1}=R\left[1+(\nabla R \cdot \nabla r) \frac{2 \epsilon}{q^{2}}\right]^{-1 / 2}=R_{0}\left[1+\epsilon\left(1-q^{-2}\right) \cos \theta\right] .
$$

The $q^{-2}$ term in this equation describes the contribution of the poloidal curvature of the field lines, which has the same direction as the curvature of the toroidal field at the outer circumference of the torus and the opposite direction at the inner one. Elongation (ellipticity) of the flux surfaces decreases the poloidal curvature near the equatorial plane and reduces the $q^{-2}$ term. 


\section{APPENDIX B: AVERAGING OF THE PARTICLE MOTION NEAR A RESONANCE}

In this Appendix we find an averaged description of the particle motion near the

resonance $\sum_{j=1}^{3} n_{j} \omega_{j}=0$, assuming that there is no other resonance relation between the frequencies of the system.

It is convenient for the foregoing to introduce the differential forms

$$
\begin{gathered}
\gamma_{g c}=\mathcal{L}_{g c} d t=\mathbf{I} \cdot d \alpha-\mathcal{E} d t \\
\delta \gamma_{2}=\delta \mathcal{L}_{2} d t=\frac{e}{c} \tilde{\mathbf{A}} \cdot d \mathbf{x}
\end{gathered}
$$

corresponding to Eqs. (53) and (32). (We restrict our consideration to the effect of the ripple and do not consider the perturbation resulting from the nonadiabaticity in the axisymmetric field, $\delta \mathcal{L}_{1}$, because, first, in this work we apply the results of this Appendix only to $\delta \mathcal{L}_{2}$ and, second, a generalization of the results to $\delta \mathcal{L}_{1}$ is evident.)

A basic idea of the averaging procedure is that "detuning" from the resonance, $\dot{\alpha}_{1}$, is small when the perturbation is small. The Euler-Lagrange equations resulting from Eq. (B1) are as follows:

$$
\begin{gathered}
\dot{\alpha}_{1}=-\left(\frac{\partial I_{2}}{\partial \mathcal{E}}\right)^{-1} \frac{\partial I_{2}}{\partial I_{1}} \equiv \Omega_{1}, \\
\dot{\alpha}_{2}=\left(\frac{\partial I_{2}}{\partial \mathcal{E}}\right)^{-1} \equiv \Omega_{2}, \\
\dot{\alpha}_{3}=-\left(\frac{\partial I_{2}}{\partial \mathcal{E}}\right)^{-1} \frac{\partial I_{2}}{\partial I_{3}} \equiv \Omega_{3},
\end{gathered}
$$

(note that we consider $I_{2}$ as a function of the other action variables and $\mathcal{E}$ ). From these equations and the smallness of $\dot{\alpha}_{1}$, we conclude that $\partial I_{2} / \partial I_{1}$ is small (and vanishes at the exact resonance). Therefore, we split $I_{2}$ into two parts, $I_{2}=I_{20}\left(I_{3}, \mathcal{E}\right)+\varepsilon I_{21}\left(I_{1}, I_{3}, \mathcal{E}\right)$, where $I_{20}\left(I_{3}, \mathcal{E}\right)$ is $I_{2}$ with the argument $I_{1}$ taken at the exact resonance, $\varepsilon$ is the ordering parameter (in the end we will set $\varepsilon=1$ to obtain physically meaningful results). The perturbation is also assumed to be $\sim \varepsilon$. We arrive at the following differential form: 


$$
\gamma=\gamma_{0}+\varepsilon \gamma_{1}
$$

where

$$
\begin{gathered}
\gamma_{0}=I_{1} d \alpha_{1}+I_{20}\left(I_{3}, \mathcal{E}\right) d \alpha_{2}+I_{3} d \alpha_{3}-\mathcal{E} d t \\
\gamma_{1}=I_{21}\left(I_{1}, I_{3}, \mathcal{E}\right) d \alpha_{2}+\frac{e}{c} \tilde{\mathbf{A}} \cdot d \mathbf{x} .
\end{gathered}
$$

An advantage of this presentation of $\gamma$ is that all slow variables $\left(I_{1}, I_{3}, \mathcal{E}\right.$, and $\left.\alpha_{1}\right)$ are constants of motion of the Euler-Lagrange equations resulting from $\gamma_{0}$.

Now we proceed to the averaging procedure. Following Ref. ${ }^{15}$, we look for a Lie transformation $\overline{\mathbf{z}}=\exp (\varepsilon \mathbf{G}) \mathbf{z}$ that makes the differential form independent on fast variables, where $\mathbf{z}=\left(\alpha_{1}, \alpha_{2}, \alpha_{3}, I_{1}, I_{3}, \mathcal{E}, t\right)$ is the vector of the variables, $\overline{\mathbf{z}}$ is the vector of the corresponding new variables free of rapid oscillations to the first order, $\mathbf{G}$ is the transformation generator. The components of the differential form $\gamma_{1}$ in the new variables look as follows: ${ }^{15}$

$$
\bar{\gamma}_{1 j}=\gamma_{1 j}-G^{i} w_{i j}+\partial_{j} S
$$

where $S$ is an arbitrary function of $\mathbf{z}$; subscripts and superscripts denote co- and contravariant components, respectively; $w_{i j}=\partial \gamma_{0 j} / \partial z_{i}-\partial \gamma_{0 i} / \partial z_{j}$ are the Lagrange brackets among the coordinates $\mathbf{z}$ in the system $\gamma_{0}$ (note that perturbations of the magnetic field modify the simplectic structure of the phase space and, in particular, the Lagrange brackets); and summation over repeating indices is implied. Then the averaging reduces to solving Eq. (B9) for $G^{i}$ and $S$, so that $\bar{\gamma}_{1 j}$ have the desired form. Specifically, we demand that all $\bar{\gamma}_{1 j}$ should vanish except for $j=\alpha_{2}$ and that $\bar{\gamma}_{1 \alpha_{2}}$ should not depend on fast variables, $\alpha_{2}$ and $\alpha_{3}$. Then the averaged Lagrangian will be given by Eq. (54), with $I\left(\bar{\alpha}_{1}, \bar{I}_{1}, \bar{I}_{3}, \mathcal{E}\right)$ to be specified. In addition, we set $G^{t}=0$ (the time variable should not be modified by the procedure).

We begin with finding $w_{i j}$. From Eqs. (B3)-(B5) one can see that all non-zero components of this matrix are

$$
w_{I_{1} \alpha_{1}}=w_{I_{3} \alpha_{3}}=w_{t \mathcal{E}}=-w_{\alpha_{1} I_{1}}=-w_{\alpha_{3} I_{3}}=-w_{\mathcal{E} t}=1,
$$




$$
\begin{gathered}
w_{\mathcal{E} \alpha_{2}}=-w_{\alpha_{2} \mathcal{E}}=\Omega_{2}^{-1}, \\
w_{\alpha_{2} I_{3}}=-w_{I_{3} \alpha_{2}}=\Omega_{2}^{-1} \Omega_{3} .
\end{gathered}
$$

On substituting $w_{i j}$ given by these equations to Eq. (B9), the $t$ component yields:

$$
G^{\mathcal{E}}=\bar{\gamma}_{1 t}=0
$$

Then we take the $\alpha_{2}$ and $\alpha_{3}$ components of Eq. (B9):

$$
\begin{gathered}
\bar{\gamma}_{1 \alpha_{2}}=\gamma_{1 \alpha_{2}}+\Omega_{2}^{-1} \Omega_{3} G^{I_{3}}-\Omega_{2}^{-1} G^{\mathcal{E}}+\partial_{\alpha_{2}} S \\
\bar{\gamma}_{1 \alpha_{3}}=\gamma_{1 \alpha_{3}}-G^{I_{3}}+\partial_{\alpha_{3}} S=0 .
\end{gathered}
$$

These equations (as well as some equations below) include the components of the differential form $\gamma_{1}$ in the $\mathbf{z}$ coordinates. By applying the chain rule to Eq. (B8), components can be expressed in terms of derivatives $\partial \mathbf{x} / \partial \mathbf{z}$, which cannot, in general, be easily calculated. Fortunately, as we will see, we will not need explicit expressions for the components of $\gamma_{1}$. It is only important for what follows that such components can be found in principle. Excluding $G^{I_{3}}$ from Eqs. (B14) and (B15), we obtain:

$$
\bar{\gamma}_{1 \alpha_{2}}=\gamma_{1 \alpha_{2}}+\Omega_{2}^{-1} \Omega_{3} \gamma_{1 \alpha_{3}}+\partial_{\alpha_{2}} S+\Omega_{2}^{-1} \Omega_{3} \partial_{\alpha_{3}} S .
$$

To solve this equation, we expand $S, \gamma_{1 \alpha_{2}}$, and $\gamma_{1 \alpha_{3}}$ in Fourier series, taking into account that the components of $\gamma$ are periodic in $\alpha_{2}$ and $\alpha_{3}$ with the period $2 n_{3} \pi$ :

$$
S=\sum_{j, k=-\infty}^{\infty} S_{j k} \exp \left(i j \frac{\alpha_{2}}{n_{3}}+i k \frac{\alpha_{2}}{n_{3}}\right)
$$

with series for $\gamma_{1 \alpha_{2}}$, and $\gamma_{1 \alpha_{3}}$ being similar. The non-oscillating terms of the equation yield

$$
\bar{\gamma}_{1 \alpha_{2}}=\frac{1}{4 n_{3}^{2} \pi^{2}} \int_{-n_{3} \pi}^{n_{3} \pi} d \alpha_{2} \int_{-n_{3} \pi}^{n_{3} \pi} d \alpha_{3}\left(\gamma_{1 \alpha_{2}}+\Omega_{2}^{-1} \Omega_{3} \gamma_{1 \alpha_{3}}\right) .
$$

To avoid the necessity to deal with separate components of $\gamma_{1}$, we introduce the family of curves $L\left(\varsigma_{10}, \alpha_{1}, P, \mu, \mathcal{E}\right)$ determined by the equations $-n_{3} \pi \leq \varsigma_{2} \leq n_{3} \pi, \varsigma_{1}=\varsigma_{10}+$ 
$\left(\omega_{1} / \omega_{2}\right) \varsigma_{2}, \varsigma_{3}=\left(\alpha_{1}-n_{1} \varsigma_{1}-n_{2} \varsigma_{2}\right) / n_{3}, P=$ const, $\mu=$ const, $\mathcal{E}=$ const. Then $\bar{\gamma}_{1 \alpha_{2}}$ can be written in the form

$$
\bar{\gamma}_{1 \alpha_{2}}=\frac{1}{4 \pi^{2} n_{3}^{2}} \int_{-n_{3} \pi}^{n_{3} \pi} d \varsigma_{10} \int_{L\left(\varsigma_{10}, \alpha_{1}, P, \mu, \mathcal{E}\right)} \gamma_{1} .
$$

Using Eqs. (B7) and (B8), we find that $I \equiv \gamma_{0 \alpha_{2}}+\bar{\gamma}_{1 \alpha_{2}}$ is given by Eqs. (55) and (56).

The oscillating terms of Eq. (B16) can be solved for $S$ :

$$
S_{j k}=i \frac{\gamma_{1 \alpha_{2}}+\Omega_{2}^{-1} \Omega_{3} \gamma_{1 \alpha_{3}}}{j+\Omega_{2}^{-1} \Omega_{3} k}
$$

where $|j|+|k|>0$. As the constant term $S_{00}$ is not essential, we may set $S_{00}=0$. Note that the absence of additional resonances is important at this point: If $\Omega_{2}^{-1} \Omega_{3}$ were rational, the denominators in some terms would vanish, and $S$ could not be found.

On finding $S$, we easily find the remaining components of $G$ from Eqs. (B15) and other components of Eq. (B9):

$$
\begin{gathered}
G^{I_{1}}=\gamma_{1 \alpha_{1}}+\partial_{\alpha_{1}} S, \quad G^{\alpha_{1}}=-\gamma_{1 I_{1}}-\partial_{I_{1}} S \\
G^{I_{3}}=\gamma_{1 \alpha_{3}}+\partial_{\alpha_{3}} S, \quad G^{\alpha_{2}}=-\Omega_{2}\left(\gamma_{1 \mathcal{E}}+\partial_{\mathcal{E}} S\right) \\
G^{\alpha_{3}}=-\gamma_{1 I_{3}}-\partial_{I_{3}} S-\Omega_{3}\left(\gamma_{1 \mathcal{E}}+\partial_{\mathcal{E}} S\right)
\end{gathered}
$$

Relations between "averaged" and "non-averaged" coordinates can be found from Eqs. (B13) and (B21)-(B23): $\overline{\mathbf{z}}=\exp (\varepsilon \mathbf{G}) \mathbf{z} \approx \mathbf{z}+\varepsilon \mathbf{G}$, where, as we have stated, $\varepsilon$ should be set to unity for physically meaningful results. 


\section{REFERENCES}

${ }^{1}$ R. J. Goldston, R. B. White, and A. H. Boozer, Phys. Rev. Lett. 47, 647 (1981).

${ }^{2}$ S. V. Putvinskiॅ, JETP Lett. 36 (1982) 397.

${ }^{3}$ A. Garren, R. J. Riddel, L. Smith, G. Bing, L. R. Henrich, T. G. Horthrop, and J. E. Roberts, in Proceedings of the 2nd United Nations International Conference on the Peaceful Uses of Atomic Energy, Geneva, 1958 (United Nations, Geneva, 1958), Vol. 31, p. 65.

${ }^{4}$ V. S. Belikov and Ya. I. Kolesnichenko, Plasma Phys. 24, 61 (1982).

${ }^{5}$ R. B. White and M. S. Chance, Phys. Fluids 27, 2455 (1984).

${ }^{6}$ R. B. White, Phys. Fluids B 2, 845 (1990).

${ }^{7}$ Ya. I. Kolesnichenko, V. S. Marchenko, and R. B. White, Phys. Plasmas 8, 3143 (2001).

${ }^{8}$ D. R. Mikkelsen, R. B. White, R. J. Akers, S. M. Kaye, D. C. McCune, and J. E. Menard, Phys. Plasmas 4, 3667 (1997).

${ }^{9}$ R. J. Hastie, G. D. Hobbs, and J. B. Taylor, in Plasma Physics and Controlled Fusion Research (International Atomic Energy Agency, Vienna, 1069), Vol. 1, p. 389.

${ }^{10}$ J. E. Howard, Phys. Fluids 14, 2378 (1971).

${ }^{11}$ R. H. Cohen, G. Rowlands, and J. H. Foote, Phys. Fluids 21, 627 (1978).

${ }^{12}$ R. Aamodt, Phys. Rev. Lett. 27, 135 (1971).

${ }^{13}$ M. N. Rosenbluth, Phys. Rev. Lett. 29, 408 (1972).

${ }^{14}$ R. G. Littlejohn, J. Plasma Phys. 29, 111 (1983).

${ }^{15}$ R. G. Littlejohn, J. Math. Phys. 23, 742 (1982).

${ }^{16}$ V. I. Arnold, Mathematical Methods of Classical Mechanics (Springer, New York, 1978).

${ }^{17}$ A. N. Kaufman, Phys. Fluids 15, 1063 (1972). 
${ }^{18}$ Ya. I. Kolesnichenko, V. V. Lutsenko, R. B. White, and Yu. V. Yakovenko, Phys. Plasmas 5, 2963 (1998).

${ }^{19}$ B. V. Chirikov, Phys. Reports 52, 265 (1979).

${ }^{20}$ A. J. Lichtenberg and M. A. Lieberman, Regular and stochastic motion (Springer-Verlag, Berlin - Heidelberg - New York, 1983).

${ }^{21}$ R. B. White, Phys. Rev. E 58, 1774 (1998).

${ }^{22}$ Yu. V. Yakovenko, in 1998 International Congress on Plasma Physics combined with 25th European Physical Society Conference on Controlled Fusion and Plasma Physics, Praha, 1998, Europhysics Conference Abstracts (The European Physical Society, PetitLancy, 1998), Vol. 22C, p. 2133.

${ }^{23}$ V. S. Belikov and Yu. V. Yakovenko, Phys. Plasmas 8, 4501 (2001).

${ }^{24}$ R. B. White, R. J. Goldston, M. Redi, and R. V. Budny, Phys. Plasmas 3, 3043 (1996).

${ }^{25}$ S. V. Putvinskii and R. V. Shurygin, Sov. J. Plasma Phys. 10, 534 (1984).

${ }^{26}$ L.-G. Eriksson and P. Helander, Nucl. Fusion 33, 767 (1993).

${ }^{27}$ Ya. I. Kolesnichenko, V. V. Lutsenko, and V. S. Marchenko, Nucl. Fusion 40, 1731 (2000).

28 J. Carlsson, Phys. Plasmas 8, 4725 (2001).

${ }^{29}$ V. A. Yavorskij, D. Darrow, V. Ya. Goloborod'ko, S. N. Reznik, U. HolzmuellerSteinacker, N. Gorelenkov, and K. Schoepf, Report P5.025 at the 28th European Physical Society Conference on Controlled Fusion and Plasma Physics, Funchal, 2001, http://www.cfn.ist.utl.pt/EPS2001/fin/pdf/P5.025.pdf.

${ }^{30}$ S. Jardin, Private communication (2001).

${ }^{31}$ O. P. Pogutse and E. I. Yurchenko, in Reviews of Plasma Physics, Vol. 11 (Consultants Bureau, N.Y., 1986), p. 65. 


\section{FIGURES}

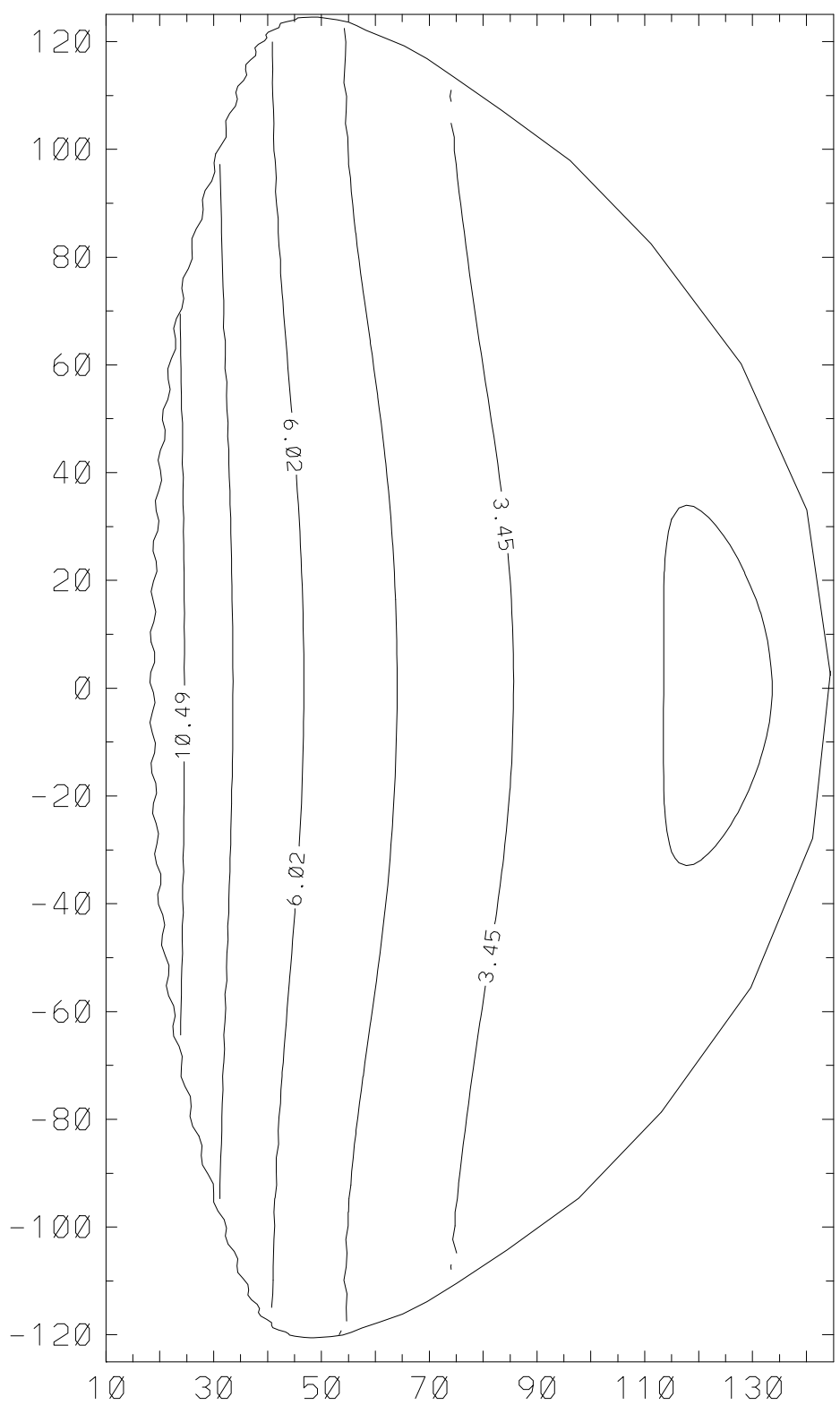

FIG. 1. Level contours of $B$ in a numerically calculated NSTX equilibrium with $\beta \approx 25 \%$. The sizes are in centimeters; $B$, in kilogausses. 


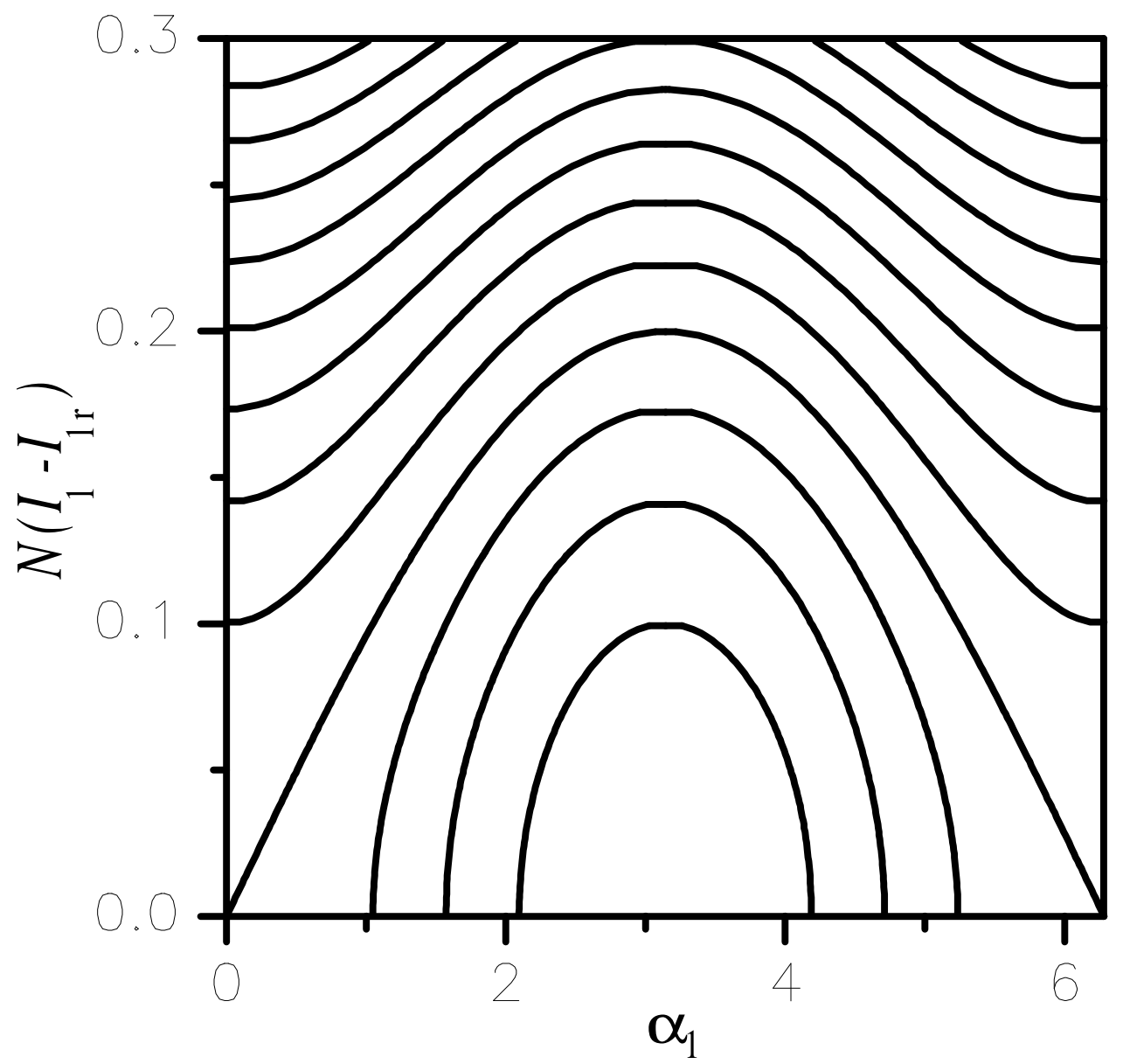

FIG. 2. Level contours of $I$ given by Eq. (64) for $Q=0.05, \varphi_{p}^{\prime}=1, N \delta I_{1}=0.01$, $\varphi_{b}=\varphi_{b}^{\prime}\left(I_{1}-I_{1 r}\right), \varphi_{b}^{\prime}=0.5$ 


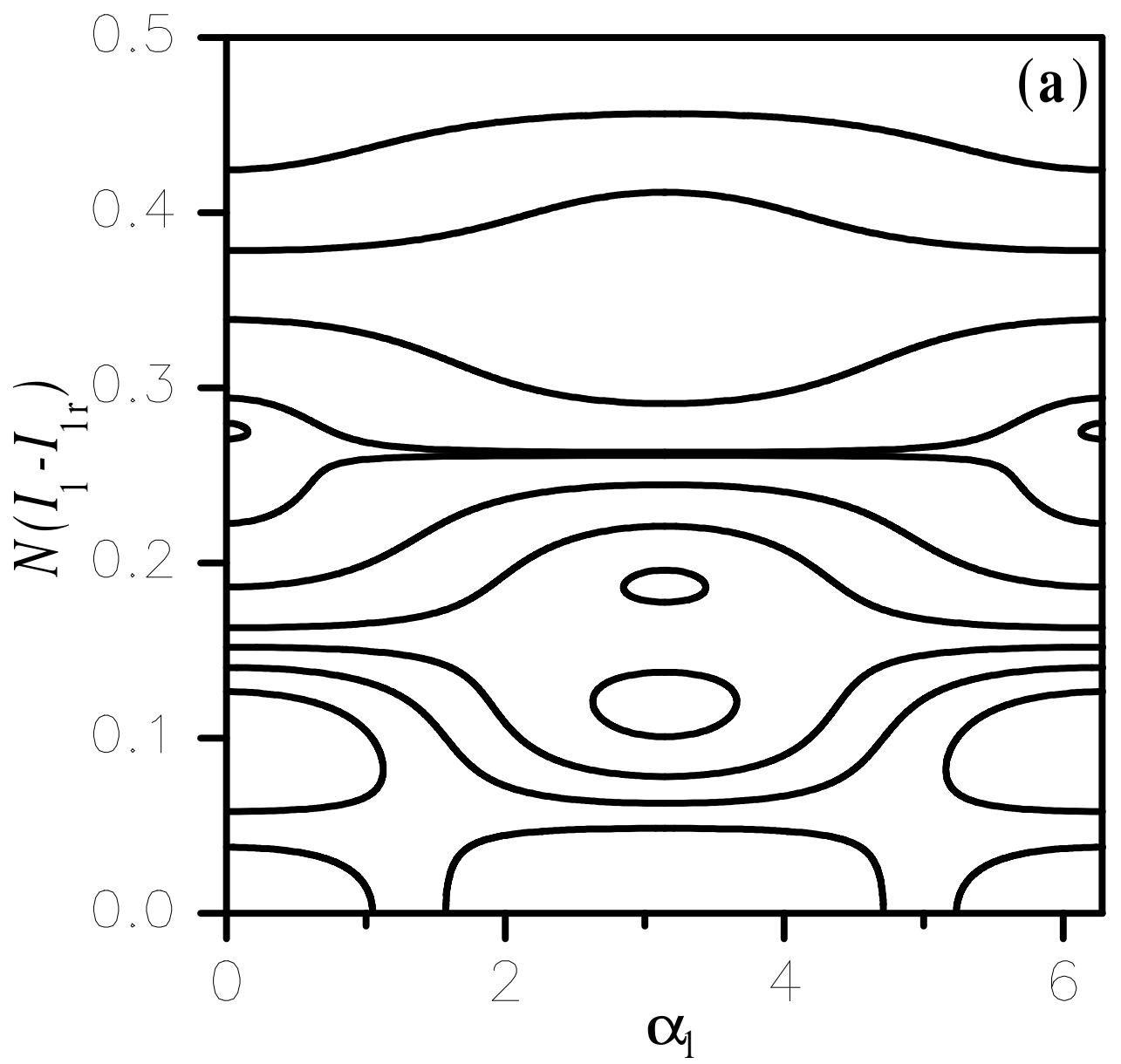




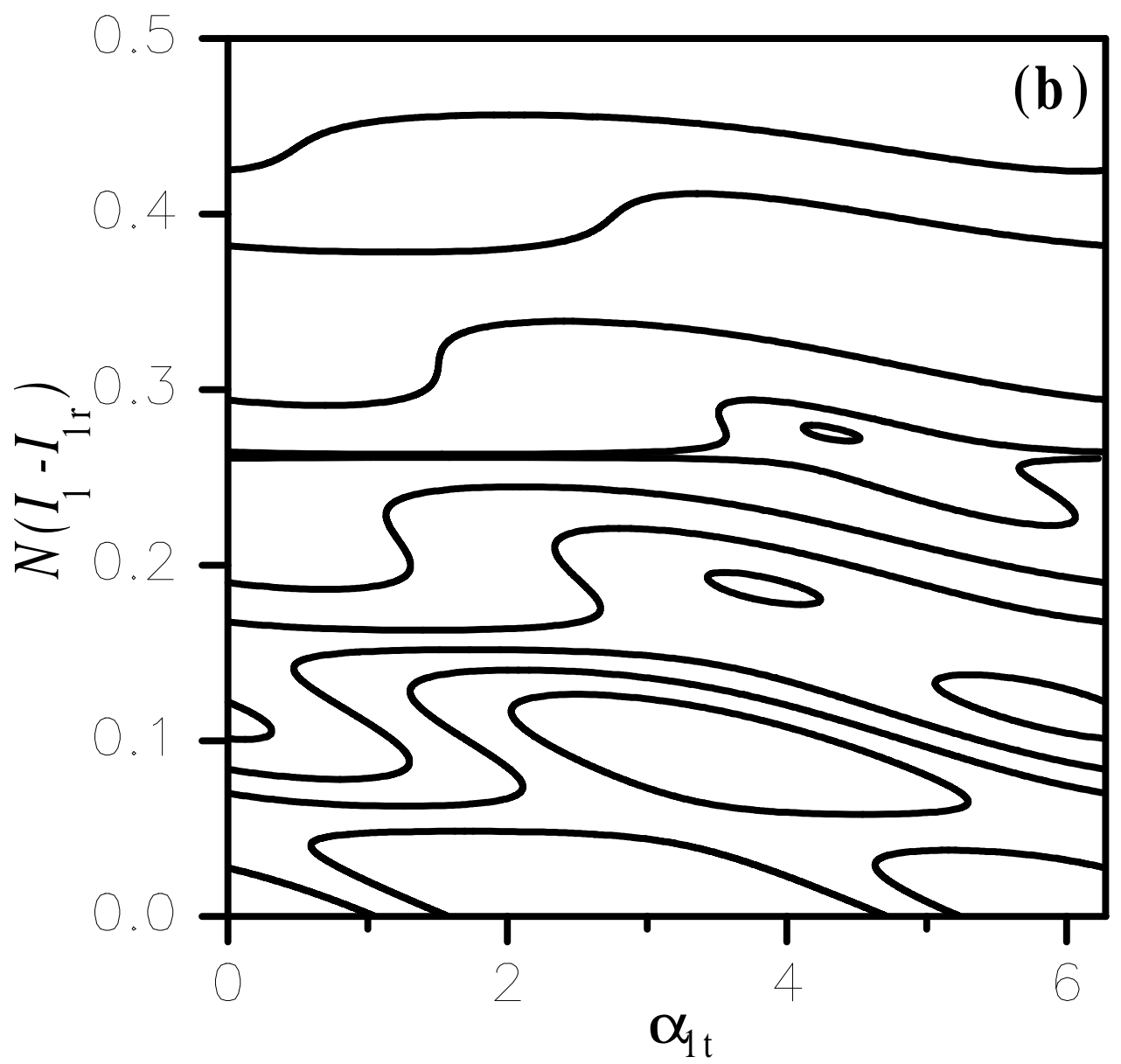

FIG. 3. The same as Fig. 2 but for $Q=6, \varphi_{b}^{\prime}=60$. (a), in the coordinates $\left(\alpha_{1}, N\left(I_{1}-I_{1 r}\right)\right)$; (b), in the coordinates $\left(\alpha_{1 t}, N\left(I_{1}-I_{1 r}\right)\right)$, where $\alpha_{1 t}=\alpha_{1}-N \phi_{b} / 2$ is the perturbation phase in one of the local resonance points. The distances between the levels are not uniform in order to show driven islands. 


\section{External Distribution}

Plasma Research Laboratory, Australian National University, Australia

Professor I.R. J ones, Flinders University, Australia

Professor J oão Canalle, Instituto de Fisica DEQ/IF - UERJ , Brazil

Mr. Gerson O. Ludwig, Instituto Nacional de Pesquisas, Brazil

Dr. P.H. Sakanaka, Instituto Fisica, Brazil

The Librarian, Culham Laboratory, England

Library, R61, Rutherford Appleton Laboratory, England

Mrs. S.A. Hutchinson, JET Library, England

Professor M.N. Bussac, Ecole Polytechnique, France

Librarian, Max-Planck-Institut für Plasmaphysik, Germany

J olan Moldvai, Reports Library, MTA KFKI-ATKI, Hungary

Dr. P. Kaw, Institute for Plasma Research, India

Ms. P.J . Pathak, Librarian, Insitute for Plasma Research, India

Ms. Clelia De Palo, Associazione EURATOM-ENEA, I taly

Dr. G. Grosso, Instituto di Fisica del Plasma, Italy

Librarian, Naka Fusion Research Establishment, J AERI, J apan

Library, Plasma Physics Laboratory, Kyoto University, J apan

Research Information Center, National Institute for Fusion Science, J apan

Dr. O. Mitarai, Kyushu Tokai University, J apan

Library, Academia Sinica, Institute of Plasma Physics, People's Republic of China

Shih-Tung Tsai, Institute of Physics, Chinese Academy of Sciences, People's Republic of China

Dr. S. Mirnov, TRINITI, Troitsk, Russian Federation, Russia

Dr. V.S. Strelkov, Kurchatov Institute, Russian Federation, Russia

Professor Peter Lukac, Katedra Fyziky Plazmy MFF UK, Mlynska dolina F-2, Komenskeho Univerzita, SK-842 15 Bratislava, Slovakia

Dr. G.S. Lee, Korea Basic Science Institute, South Korea

Mr. Dennis Bruggink, Fusion Library, University of Wisconsin, USA

Institute for Plasma Research, University of Maryland, USA

Librarian, Fusion Energy Division, Oak Ridge National Laboratory, USA

Librarian, Institute of Fusion Studies, University of Texas, USA

Librarian, Magnetic Fusion Program, Lawrence Livermore National Laboratory, USA

Library, General Atomics, USA

Plasma Physics Group, Fusion Energy Research Program, University of California at San Diego, USA

Plasma Physics Library, Columbia University, USA

Alkesh Punjabi, Center for Fusion Research and Training, Hampton University, USA

Dr. W.M. Stacey, Fusion Research Center, Georgia Institute of Technology, USA

Dr. J ohn Willis, U.S. Department of Energy, Office of Fusion Energy Sciences, USA

Mr. Paul H. Wright, Indianapolis, Indiana, USA 
The Princeton Plasma Physics Laboratory is operated by Princeton University under contract with the U.S. Department of Energy.

\author{
Information Services \\ Princeton Plasma Physics Laboratory \\ P.O. Box 451 \\ Princeton, NJ 08543
}

Phone: 609-243-2750

Fax: 609-243-2751

e-mail: pppl_info@pppl.gov

Internet Address: http://www.pppl.gov 